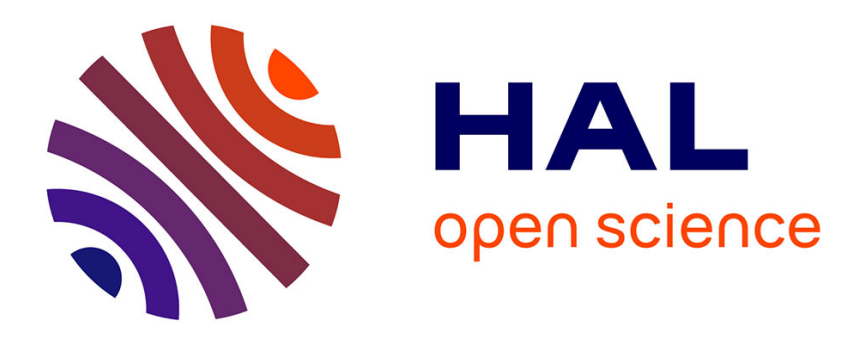

\title{
Applications des lasers picosecondes à l'optoélectronique
}

J. Chesnoy

\section{To cite this version:}

J. Chesnoy. Applications des lasers picosecondes à l'optoélectronique. Revue de Physique Appliquée, 1989, 24 (5), pp.577-596. 10.1051/rphysap:01989002405057700 . jpa-00246083

\section{HAL Id: jpa-00246083 https://hal.science/jpa-00246083}

Submitted on 1 Jan 1989

HAL is a multi-disciplinary open access archive for the deposit and dissemination of scientific research documents, whether they are published or not. The documents may come from teaching and research institutions in France or abroad, or from public or private research centers.
L'archive ouverte pluridisciplinaire HAL, est destinée au dépôt et à la diffusion de documents scientifiques de niveau recherche, publiés ou non, émanant des établissements d'enseignement et de recherche français ou étrangers, des laboratoires publics ou privés. 
Classification

Physics Abstracts

$42.60 \mathrm{~F}-42.60 \mathrm{~K}$

\title{
Applications des lasers picosecondes à l'optoélectronique
}

\author{
J. Chesnoy \\ Laboratoire d'Optique Quantique, Ecole Polytechnique, 91128 Palaiseau Cedex, France \\ Laboratoires de Marcoussis, Route de Nozay, 91460 Marcoussis, France
}

(Reçu le 31 octobre 1988, révisé le 16 janvier 1989, accepté le 6 février 1989)

Article de mise au point

Résumé. - Cet article tend à dégager parmi les techniques laser ultra-rapides (picosecondes et femtosecondes) celles qui devraient être appelées à un développement dans le domaine de l'électronique, de l'optoélectronique et des télécommunications. Sont d'abord analysées les possibilités d'étude de circuits électroniques ultrarapides par laser picoseconde. Les possibilités d'étude de circuits optoélectroniques sont ensuite considérées ainsi que le développement d'appareillages possibles dans ce domaine. Les applications potentielles des lasers à impulsions brèves aux télécommunications à haut débit sont aussi analysées et l'accent est finalement mis sur les éventuels développements dans le domaine de l'optoélectronique hyperfréquence. Le développement de sources picosecondes compactes et simples, probablement à base de diodes laser, amorcerait l'expansion industrielle de ces techniques nouvelles.

\begin{abstract}
This paper tends to bring out, between ultrafast laser techniques (picosecond and femtosecond), those which should emerge in the domains of electronics, optoelectronics and telecommunications. First, the possibility to study ultrafast electronic circuits using picosecond lasers is analysed. A similar application to optoelectronic devices is then considered as well as the development of systems for diagnostics in this domain, based on ultrashort light pulses techniques. The potential applications of picosecond and femtosecond lasers to high rate telecommunications are finally envisaged, with an accent on the eventual developments in the domain of microwave optoelectronics. We underline the interest of developing compact and safe picosecond laser sources, probably based on laser diodes, to initiate industrial expansion of these new techniques.
\end{abstract}

\section{Introduction.}

La création d'impulsions de très courtes durées par couplage des modes longitudinaux d'un laser est déjà une technique ancienne, traitée dans de nombreux ouvrages de référence $([1,2]$ par exemple). Les années récentes ont vu des progrès techniques importants qui ont permis de passer du domaine des « picosecondes » $\left(10^{-12} \mathrm{~s}\right)$ à celui des « femtosecondes » (avec 6 femtosecondes comme plus faible durée atteinte [3]). Les techniques de mesures en régime picoseconde sont maintenant facilitées par le remplacement des lasers de puissance à basse cadence de répétition par des sources quasi continues ou à haute cadence $(>1 \mathrm{kHz})$ qui permettent une amélioration considérable de la sensibilité de détection. Les possibilités actuelles de mesure de phénomènes ultra-rapides permettent aux lasers picosecondes d'amorcer une sortie des laboratoires spécialisés vers les laboratoires industriels. Parmi les domaines d'application, on observe un essor des mesures physiques appliquées à l'électronique et à l'optoélec- tronique rapides pour lesquelles une mise au point sera présentée dans la suite.

Les particularités du couplage de modes d'un laser, de la propagation des impulsions brèves et des techniques de mesure dans ce domaine vont être rappelées tout d'abord rapidement, avant d'en présenter de manière détaillée les applications les plus prometteuses.

1. CRÉation ET PROPAgation DES impulsions LUMINEUSES BRÈVES. - Parmi les techniques permettant de générer des impulsions lumineuses brèves, le couplage de modes reste l'une des plus simples et la plus efficace. Un laser est dit à modes couplés quand il oscille sur plusieurs modes longitudinaux mis en phase, de sorte que le champ électrique de l'onde lumineuse soit rayonné sous la forme d'une somme sur $m$ modes :

$$
E(t)=E_{0} \mathrm{e}^{i \omega_{0} t} \sum_{m} \mathrm{e}^{i m \Delta \omega t}+\text { c.c. }
$$

où $\omega_{0}$ est la fréquence optique centrale et $\Delta \omega$ l'écart 
de fréquence entre modes : $\Delta \omega=2 \pi \nu$ (où $\nu$ est la fréquence d'aller et retour de la lumière dans la cavité optique). Un laser à modes couplés émet ainsi de manière quasi stationnaire un train d'impulsions brèves espacées d'un aller et retour dans la cavité. La durée des impulsions vaut $1 / \nu M$, où $M$ est le nombre de modes couplés rayonnés.

Deux propriétés des lasers à modes couplés peuvent être utilisées, d'une part la courte durée des impulsions rayonnées, mise à profit pour étudier la dynamique d'événements ultra-rapides, et d'autre part la cadence de répétition régulière des impulsions, utilisable par exemple pour les télécommunications optiques. Les lasers à modes couplés permettent d'obtenir des impulsions lumineuses plus courtes par plusieurs ordres de grandeur que les impulsions les plus brèves disponibles électroniquement.

Le couplage de modes peut être obtenu sur un grand nombre de lasers. Les milieux lasers les plus utilisés sont à base de solides (YAG ou verre dopé au néodyne) ou de colorants, pour lesquels des systèmes commerciaux sont disponibles. Le développement des lasers à semi-conducteurs est prometteur mais moins avancé. Ces lasers donnent une possibilité supplémentaire d'obtenir des impulsions lumineuses brèves par commutation du gain $[4,5]$. Ces sources devraient prendre une importante grandissante vu leur simplicité [6].

Les impulsions lumineuses brèves sont larges en fréquence, ce qui affecte la propagation linéaire de ces impulsions. A énergie transportée donnée, elles sont plus intenses que des impulsions plus longues et vont ainsi manifester rapidement des comportements de propagation non linéaire. Enfin, quand la durée des impulsions devient plus brève que le temps de réponse d'un processus dynamique du matériau optique traversé, le régime transitoire de la propagation doit être considéré.

L'indice de réfraction $n$ d'un milieu optique détermine la vitesse de propagation linéaire d'une impulsion lumineuse. La vitesse de groupe $V_{\mathrm{g}}$ vaut $c /(n-\lambda \mathrm{d} n / \mathrm{d} \lambda)$ à la longueur d'onde centrale $\lambda \mathrm{de}$ l'impulsion. Si cette vitesse de groupe n'est pas constante sur la largeur spectrale de l'impulsion, la dispersion des vitesses de groupe amène un élargissement temporel au cours de la propagation. Une impulsion brève de durée $\tau_{\mathrm{p}}$ étant nécessairement large en fréquence $\left(\Delta \nu \geqslant 1 / \tau_{\mathrm{p}}\right)$, la distance de propagation sans déformation est limitée par cet effet. Ainsi, une impulsion de $100 \mathrm{fs}$ dans le visible est sensiblement déformée par la propagation dans quelques centimètres de verre. Cet effet devient dramatique pour les impulsions les plus brèves actuellement produites, car l'allongement temporel relatif $\Delta \tau_{\mathrm{p}} / \tau_{\mathrm{p}}$ est proportionnel à $\Delta \nu^{2}\left(\right.$ ou $\left.1 / \tau_{\mathrm{p}}^{2}\right)$, ce qui limite l'utilisation de telles impulsions. Pour des impulsions lumineuses plus longues, de l'ordre de la picoseconde, la dispersion des vitesses de groupe devient sans conséquence dans la pratique, sauf après de grandes distances de propagation. Ainsi, une impulsion de 10 ps se propageant dans une fibre optique de dispersion valant $15 \mathrm{ps} / \mathrm{nm} . \mathrm{km}$ est allongée d'un facteur 2 après $7 \mathrm{~km}$. Bien que cet effet soit beaucoup moins important que pour une impulsion électrique se propageant sur une ligne conductrice, il apporte une limite aux propagations d'impulsions optiques brèves à grande distance (par exemple pour des applications de télécommunications à très haute cadence).

Des effets optiques non linéaires peuvent être atteints pour des impulsions brèves à de faibles niveaux d'énergie. Ainsi, des impulsions femtosecondes d'une fraction de microjoules peuvent être converties en fréquence efficacement par des effets non linéaires comme le doublement de fréquence, l'effet paramétrique ou l'automodulation de phase [7]. Par contre, les phénomènes impliquant l'excitation résonnante d'un milieu matériel deviennent peu efficaces, voire impossibles à l'aide d'impulsions lumineuses brèves par rapport au temps de réponse du milieu [8]. Tel est le cas des effets Brillouin et Raman stimulés qui deviennent inaccessibles aux impulsions picosecondes, même à des niveaux de puissance très supérieurs aux seuils en régime stationnaire excepté dans les fibres optiques où les grandes distances de propagation permettent d'atteindre facilement le seuil de la diffusion Raman stimulée. De même, les seuils de dommage optique, impliquant des phénomènes catastrophiques lents, sont notablement augmentés en régime d'impulsions brèves, ce qui simplifie la manipulation des impulsions intenses. Le premier phénomène optique non linéaire à apparaître en régime picoseconde est souvent l'automodulation de phase qui élargit spectralement les impulsions et s'accompagne spatialement de l'autofocalisation du faisceau lumineux [7]. C'est le phénomène limitant l'utilisation des impulsions brèves intenses. En contrepartie, cet effet donne une possibilité de compression temporelle des impulsions lumineuses par « fibre et réseaux » $[9,3$, 10].

2. TECHNIQUES DE MESURE EN RÉGIME PICOSECONDE ET DOMAINES D'APPLICATION. - L'électronique actuelle ne permet pas de disposer d'appareils de mesure ayant une résolution meilleure qu'une fraction de nanoseconde, sauf dans les deux cas de l'oscilloscope à échantillonnage et de la caméra à balayage de fentes (voir plus loin). Les lasers picosecondes restent les seuls moyens de mesure des événements rapides inaccessibles par les techniques électroniques.

Le principe des mesures utilisant des impulsions lumineuses brèves est essentiellement basé sur l'échantillonnnage optique. Ces techniques d'« excitation-sondage » (Fig. 1) décomposent la mesure en 


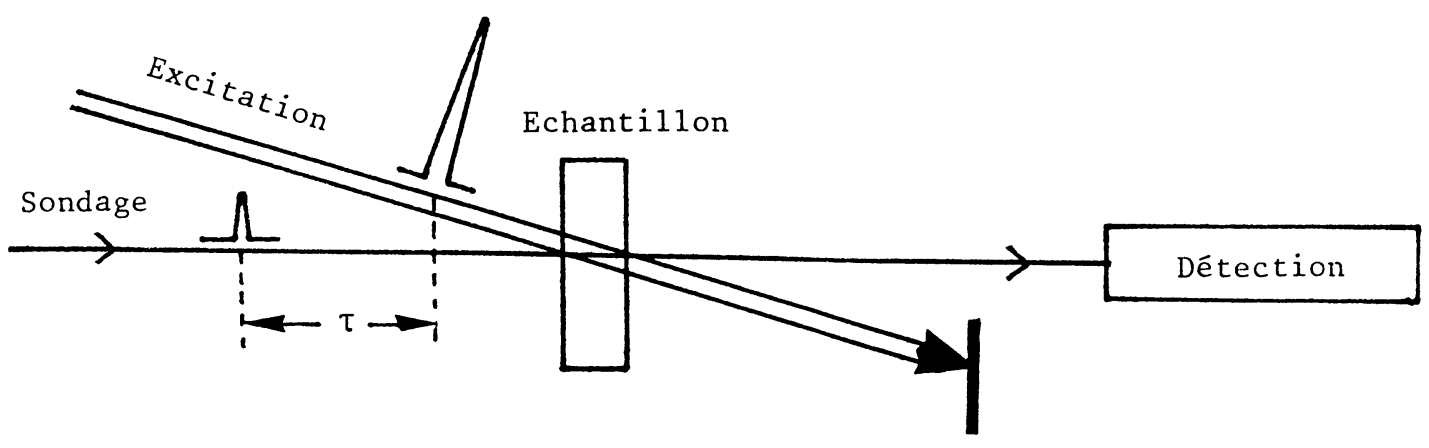

Fig. 1. - Schéma d'une expérience d'excitation-sondage. Une impulsion laser ultra-brève intense excite l'échantillon. Ensuite, une deuxième impulsion, retardée d'un délai $\tau$ variable, sonde le retour à l'équilibre d'une propriété optique mesurée par un système de détection. La résolution temporelle de cet échantillonnage optique est donnée par la durée des impulsions lumineuses.

[Principle of an excite and probe measurement. An intense ultrashort light pulse excites the sample. Then, a weak pulse probes after a variable delay the decay of an optical property measured by a detection set-up. The temporal resolution of this optical sampling is determined by the duration of the light pulses.]

deux étapes: une première impulsion lumineuse intense excite le système à étudier, et une deuxième impulsion de faible intensité est envoyée après un délai $\tau$ pour lire une propriété optique permettant de suivre le retour à l'équilibre du système. La mesure complète est effectuée en variant le délai temporel $\tau$ entre excitation et sondage. La mesure détermine ainsi par échantillonnage la «fonction réponse » du système (soumis à une excitation infiniment brève) avec une résolution temporelle déterminée par la durée des impulsions lumineuses utilisées (le plus souvent, la fonction d'appareil est une fonction de corrélation des intensités en présence). Certaines variantes techniques permettent une capture du signal en un seul coup laser, donc une analyse en temps réel du phénomène étudié et non plus en « temps équivalent». La configuration la plus simple est la mesure de durée d'impulsions lumineuses en un seul tir laser $[11,12]$, basée sur la variation dans l'espace du délai temporel. Le même principe a été utilisé très tôt pour des mesures sur des systèmes physiques variés [1]. D'autres possibilités s'offrent aussi d'utiliser à la fois la résolution spectrale et temporelle des impulsions [1, 13].

Ces techniques d'analyse des phénomènes temporels à l'échelle picoseconde ou femtoseconde ont été appliquées dans les domaines scientifiques les plus variés. Le tableau I donne des ordres de grandeur des temps caractéristiques associés à des phénomènes physiques accessibles aux techniques d'excitation-sondage. Un grand nombre d'exemples pourra être trouvé dans la bibliographie [1] et dans la série des comptes rendus de conférences «Ultrafast Phenomena " (Springer Ser. Chem. Phys., Vols. 14, 23, 38, 46 et 48), ainsi que dans des numéros spéciaux de revues $[14,15]$.

La possibilité d'analyse d'un phénomène ultrarapide est déterminée par l'accès optique à ce
Tableau I. - Quelques exemples des échelles de temps impliquées dans des processus physiques, physico-chimiques ou biologiques élémentaires, qui sont l'objet d'études par des techniques optiques basées sur les lasers picosecondes ou femtosecondes.

[Several examples of the time scales involved in physical, chemical and biological elementary processes, are given. They are the object of studies by optical techniques based on picosecond and femtosecond lasers.]

\begin{tabular}{|l|r|}
\cline { 2 - 2 } \multicolumn{1}{c|}{} & \multicolumn{1}{c|}{$\begin{array}{c}\text { Echelle } \\
\text { de temps } \\
\text { impliquée }\end{array}$} \\
\hline $\begin{array}{l}\text { Processus physiques : } \\
\text { Rotation moléculaire en phase liquide } \\
\text { Vibration moléculaire }\end{array}$ & $\begin{array}{r}1-100 \mathrm{ps} \\
\text { Transferts électrons-réseau (dans un } \\
\text { semi-conducteur ou dans un métal) }\end{array}$ \\
Temps de collision d'électrons & $100 \mathrm{fs}-10 \mathrm{ps}$ \\
Temps de fluorescence électronique & $\sim 100 \mathrm{fs}$ \\
(molécules ou semi-conducteurs) & $\leqslant 100 \mathrm{fs}$ \\
Processus chimiques ou biochimiques : & $100 \mathrm{ps}-10 \mathrm{~ns}$ \\
Transfert intermoléculaire d'énergie & $100 \mathrm{fs}-10 \mathrm{~s}$ \\
Equilibration structurale d'une molécule & $100 \mathrm{fs}-10 \mathrm{ps}$ \\
Réarrangement d'un solvant & $100 \mathrm{fs}-50 \mathrm{ps}$ \\
Relaxation diélectrique & $1-100 \mathrm{ps}$ \\
Processus élémentaires de la vision & $0,5-5 \mathrm{ps}$ \\
Processus élementaires de la photosynthèse & $1-10 \mathrm{ps}$ \\
\hline
\end{tabular}

phénomène. C'est ainsi que les possibilités d'étudier la dynamique des phénomènes optiques ont été d'abord exploitées pour étudier par exemple la dynamique de propriétés optiques en physique et plus récemment les processus élémentaires de la vision en biologie [16]. L'intérêt technologique des études possibles de circuits électroniques ultra-rapides traitées dans la suite s'est concrétisé grâce au 
développement d'interfaces optoélectroniques entre le laser picoseconde et l'électronique à étudier. Les possibilités d'application aux télécommunications seront aussi abordées en soulignant que la cadence de répétition des impulsions, plus que leur durée, est alors exploitée, ouvrant à la limite des possibilités dans le domaine des hyperfréquences.

\section{Apport à l'électronique et à l'optoélectronique rapi- des.}

L'électronique rapide est promise à un progrès important lors des prochaines années afin de répondre aux besoins des télécommunications optiques, des transmissions hyperfréquences et de l'informatique : les liaisons de télécommunications optiques actuellement commercialisées ont une capacité maximale voisine de 2 Gbits/s, mais les systèmes développés en laboratoire permettent d'atteindre une cadence de 5-10 Gbits/s, ce qui singifie que chacun des composants doit avoir une bande passante de l'ordre de $10 \mathrm{GHz}$ (soit un temps de montée inférieur à $30 \mathrm{ps}$ ). Les télécommunications hyperfréquence tendent à utiliser au mieux les bandes HF pour répondre d'abord à des besoins militaires ou spatiaux. La miniaturisation des composants pour radars ou antennes est aussi l'objet de recherches actives. Finalement, la rapidité qui sera demandée aux systèmes informatiques est sans limite puisqu'on voit déjà des ordinateurs de la nouvelle génération utiliser de l'électronique refroidie, à base de semiconducteurs III-V.

Les techniques classiques de test de circuits électroniques poussées à leurs limites ne permettent pas toujours de caractériser un circuit donné. Souvent, les composants rapides sont soumis essentiellement à des tests à basse fréquence et le comportement à haute fréquence est seulement estimé. Ce test rudimentaire deviendra impossible si les couplages capacitifs entre circuits se généralisent (le circuit n'est alors opérationnel qu'à haute fréquence). Dans la suite, les possibilités de tester des circuits rapides par laser vont être analysées.

Les possibilités d'étude et de test de circuits optoélectroniques apparaissent comme une application naturelle de techniques utilisant des impulsions lumineuses brèves, dès lors que la présence du port d'entrée ou de sortie optique permet une simplification des techniques décrites précédemment pour l'étude de circuits purement électroniques. Je donnerai comme exemple les possibilités d'étude de composants élémentaires comme les photodiodes rapides et les lasers à semi-conducteurs.

\section{A. TeChNiques Classiques DE TEST DE CIRCUITS ÉLECTRONIQUES.}

1. Analyse électrique temporelle. — Si l'on considère un circuit linéaire sur lequel on entre un signal $v_{\mathrm{c}}(t)$, le signal de sortie $v_{\mathrm{s}}(t)$ est complètement déterminé par la fonction réponse $R(t)$ du système, définie comme la valeur prise par $v_{\mathrm{s}}(t)$ pour une excitation instantanée $v_{\mathrm{e}}(t)=\delta(t)$. Alors, de façon générale :

$$
\begin{aligned}
v_{\mathrm{s}}(t) & =\int R\left(t-t^{\prime}\right) v_{\mathrm{e}}\left(t^{\prime}\right) \mathrm{d} t^{\prime} \\
& =R(t) \otimes v_{\mathrm{e}}(t)
\end{aligned}
$$

Cette expression peut être généralisée au cas de circuits non linéaires où la réponse $R$ devient une fonction de plusieurs variables temporelles [17].

Mesure et limitation. - L'étude du circuit consiste à le soumettre à une excitation $v_{\mathrm{e}}(t)$ aussi brève que possible $(\sim \delta(t))$ et à détecter $v_{\mathrm{s}}(t)$, le plus souvent en utilisant un oscilloscope à échantillonnage.

D'une part, la durée de l'impulsion d'entrée est limitée à $60 \mathrm{ps}(\sim 6 \mathrm{GHz})$ pour les génératéurs actuels basés sur des diodes rapides (« step recovery diodes ») et d'autre part, la tension est limitée à $5 \mathrm{~V}$. Une excitation en créneau du circuit peut être plus rapide $(20 \mathrm{ps})$ en utilisant des diodes tunnel qui sont cependant limitées à $0,25 \mathrm{~V}$. D'autre part, pour la détection, les têtes à échantillonnage commerciales actuelles sont limitées à $25-30 \mathrm{ps}$ de temps de montée $(12 \mathrm{GHz})$.

Ainsi, une étude temporelle d'un circuit peut difficilement avoir une résolution temporelle meilleure que 25-30 ps, soit une limite en fréquence de $12 \mathrm{GHz}$. A noter cependant que des oscilloscopes à échantillonnage vont être commercialisés aveć une résolution de $15 \mathrm{ps}$ et peut-être de $8 \mathrm{ps}$ (Colby Instruments, USA), tandis qu'un matériel refroidi à l'hélium liquide et atteignant 5 ps de résolution est aussi sur le marché (Hypres, USA). Les supraconducteurs à haute température provoqueront la renaissance de l'électronique à jonctions Josephson, à l'aide de laquelle des têtes à échantillonnage pourraient en principe atteindre quelques picosecondes de résolution [18].

Une limitation de cette technique de test est aussi son incapacité à permettre l'analyse du circuit point par point afin d'étudier indépendamment les divers éléments. En effet, l'adaptation $50 \Omega$ est indispensable à cette méthode de mesure si l'on veut préserver la résolution temporelle : seule la sortie du circuit peut être analysée, ou éventuellement une sortie intermédiaire prévue dès la conception du circuit.

2. Analyse électrique en fréquence. - Lors de l'analyse en fréquence d'un circuit, on injecte en entrée un signal sinusoïdal à la fréquence $\omega$

$$
v_{\mathrm{e}}(t) \sim v_{0} \cos \omega t
$$

En sortie, un signal est détecté :

$$
v_{\mathrm{s}}(t)=|R(\omega)| v_{0} \cos (\omega t+\varphi(\omega)) .
$$


La fonction complexe $R(\omega)=|R(\omega)| \mathrm{e}^{i \varphi(\omega)}$ est une donnée strictement équivalente à la connaissance de la fonction $R(t)$, car elles sont reliées par la Transformée de Fourier :

$$
R(\omega)=\int \mathrm{d} t \mathrm{e}^{-i \omega t} R(t)
$$

Mesure et limitation. - L'analyseur de spectre donne accès à $|R(\omega)|$. Cette donnée incomplète est suffisante si le circuit est destiné à être excité en régime sinusoïdal. L'analyseur de réseaux donne accès à la réponse en fréquence $R(\omega)$ avec détermination de la phase $\varphi(\omega)$.

Ces méthodes, qui bénéficient du développement des techniques hyperfréquence, permettent l'analyse des circuits jusqu'à des fréquences de plusieurs centaines de gigahertz, limitées en fait à des fréquences nettement inférieures à $100 \mathrm{GHz}$ par les boîtiers, connecteurs et câbles. Il existe la possibilité aussi d'effectuer une mesure temporelle et d'en prendre la Transformée de Fourier [19].

La méconnaissance de la phase par l'analyseur de spectre n'est pas satisfaisante si le circuit est destiné à être utilisé en régimes d'impulsions. L'analyseur de réseaux est un appareillage lourd pour lequel l'incertitude sur les phases n'est jamais complètement levée aux plus hautes fréquences. En cas de non-linéarité d'un circuit (digital), une modélisation complexe est nécessaire pour accéder au comportement en régime d'impulsions.

Les possibilités de test sont encore limitées par la nécessaire adaptation $50 \Omega$ de la sortie utilisée, ce qui rend difficile l'accès à des points quelconques du circuit. Ces techniques nécessitent la pose de pointes de test et deviennent délicates avec la miniaturisation des circuits intégrés. Une solution est apportée par l'utilisation du sondage par faisceau d'électrons à l'aide d'un microscope électronique à balayage [20, 21].

B. ÉCHANTILLONNAGE OPTIQUE DE CIRCUITS PAR LASERS PICOSECONDES. - Deux besoins principaux ont poussé le développement des techniques de sondage de circuits électroniques par lasers picosecondes : l'amélioration de la résolution temporelle et la possibilité de résoudre spatialement, point par point, le comportement d'un circuit éventuellement sans contact et sans contrainte d'adaptation d'impédance.
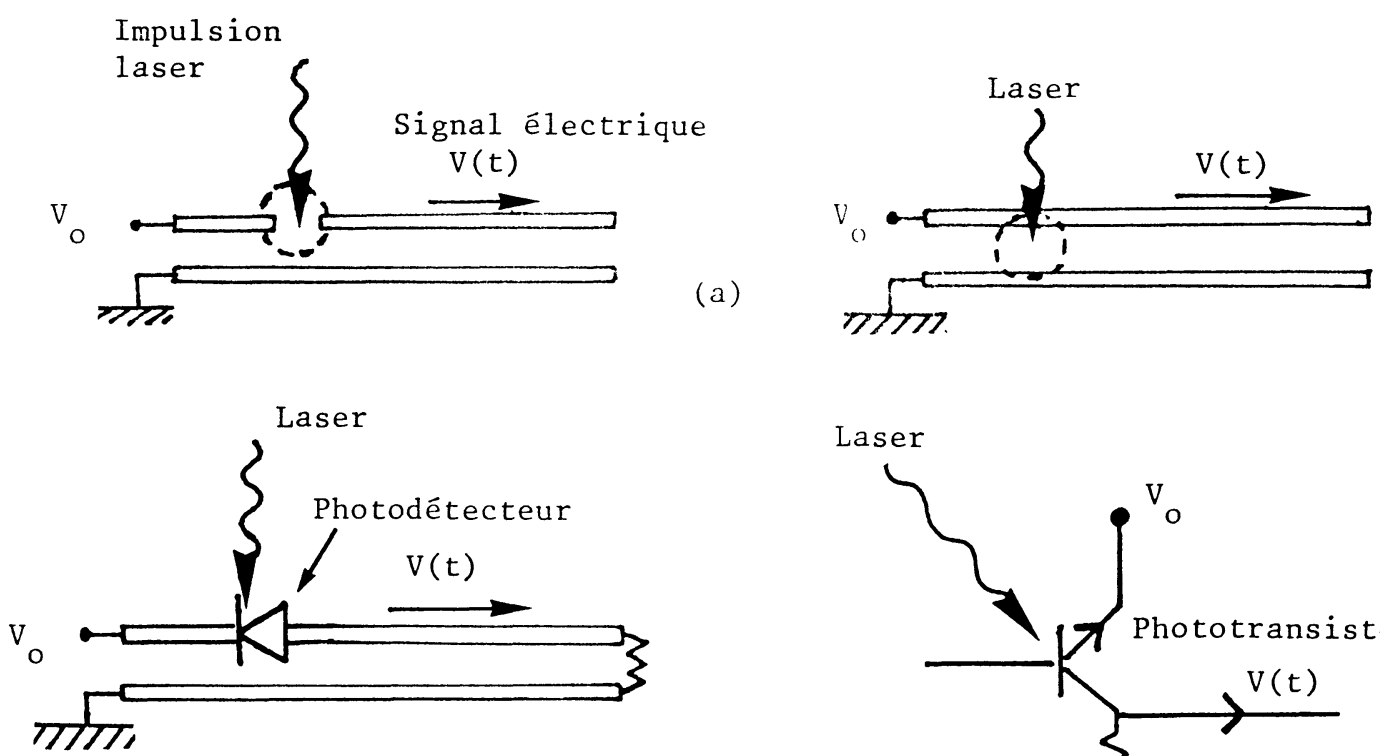

(b)

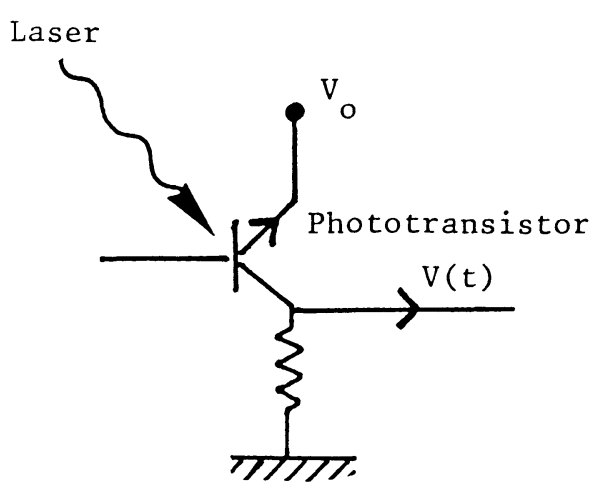

(c)

Fig. 2. - Création d'une impulsion électrique brève par laser. Une impulsion électrique peut être générée sur une ligne électrique par effet photoconducteur à l'aide d'une impulsion laser picoseconde (a). Deux exemples de géométrie sont indiqués. En (b), une possibilité d'utiliser un détecteur photovoltaïque est aussi illustrée, tandis qu'en (c), l'impulsion électrique est créée par illumination d'un phototransistor. Ces deux dernières techniques sont plus simples mais moins performantes.

[Production of a short electric pulse by a laser pulse. An electric pulse can be generated on a conducting line by picosecond photoconductivity. Two possible geometries are illustrated (a). In (b), a photovoltaic detector is used, while in (c), the electric pulse is created by picosecond illumination of a phototransistor. These two last techniques are more simple but less performant.] 
Les possibilités d'échantillonnage de circuits électroniques reposent sur les possibilités d'exciter un circuit par laser et de détecter des tensions par laser.

1. Excitation de circuits par laser (Fig. 2). - Le «switch» photoconducteur [22-24] fait passer un «gap » de ligne électrique de l'état isolant à l'état conducteur par les porteurs créés par l'impulsion lumineuse. Le temps de montée de la conductance est donné par la durée de l'impulsion lumineuse, le temps de descente par la durée de vie des porteurs qui peut être raccourcie par dopage du photoconducteur $\left(\mathrm{O}^{+} / \mathrm{Si}-\mathrm{Cr} / \mathrm{GaAs}\right)$. L'association de deux « switches " permet aussi de contrôler indépendamment la commutation «on " et "off » [25]. La géométrie de la ligne électrique détermine les possibilités temporelles ultimes de cette génération d'impulsions électriques [26-28]. Des impulsions électriques subpicosecondes peuvent maintenant être créées et propagées par cette technique $[29,30]$. De

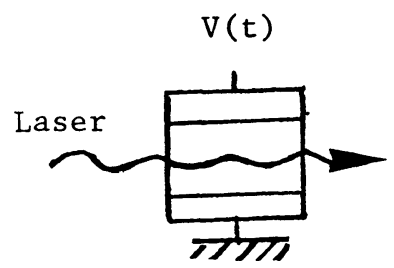

(a) SONDAGE

ELECTRO-OPTIQUE

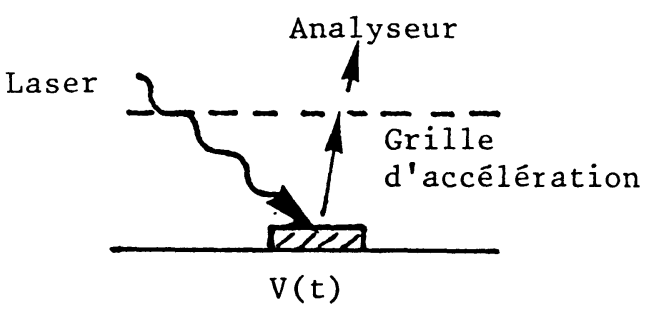

(c)

SONDAGE PHOTOEMISSIF très hautes tensions (plusieurs kilovolts) ont pu être commutées par ailleurs [31]. Il a été souligné que de tels « switches » photoconducteurs pouvaient constituer l'élément de synchronisation du laser picoseconde avec une porte optique [32] ou avec un appareil électronique [33].

Une photodiode peut être naturellement utilisée pour convertir un signal lumineux en signal électrique avec une très grande sensibilité. La rapidité de commutation est limitée par la capacité de la jonction. Seules de faibles tensions pourront être commutées par ce moyen.

Les phototransistors permettent une conversion optique/électrique avec amplification du signal électrique. La rapidité intrinsèque du transistor limite la durée minimale accessible, ce qui donne par ailleurs un moyen d'étudier la réponse du transistor luimême [34]. L'intérêt de cette méthode d'excitation électrique réside dans le fait que chaque transistor d'un circuit est un phototransistor dont la base peut

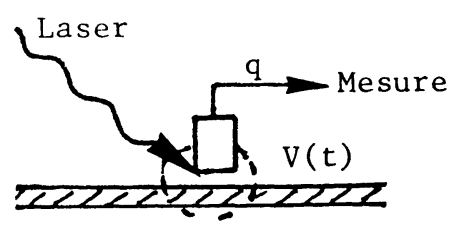

(b)

SONDAGE PHOTOCONDUCTEUR

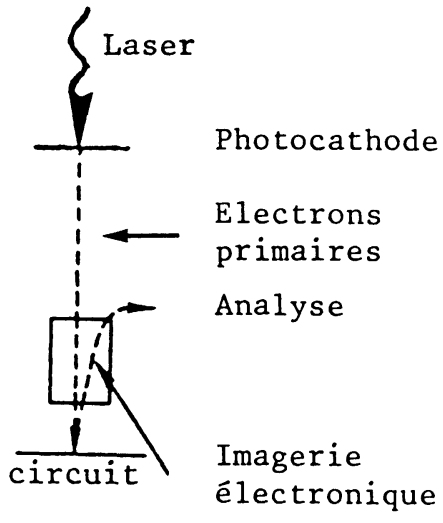

(d) ELECTRONIQUE

Fig. 3. - Sondage d'une tension électrique par laser. Un champ électrique induit dans un matériau électro-optique une biréfringence qui peut être lue par laser (a). En (b), un «switch » photoconducteur fait passer une charge $q$ sur la ligne de mesure en présence de l'impulsion laser de sonde et cette charge est proportionnelle à $V(t)$. En (c), l'effet photoémissif est utilisé et la vitesse des électrons après accélération permet de connaître la tension $V(t)$ du conducteur émetteur. En (d), le circuit est analysé par imagerie électronique donnée par un faisceau pulsé obtenu par l'éclairement d'une photocathode.

[Laser probing of an electrical voltage. The electric field induces in an electro-optic material a birefringence that can be probed by a laser pulse (a). In (b), an electrical charge proportional to the voltage $V(t)$ is taken off by the photoconductive switch and measured [45]. The photoemissive effect is used in (c) and the velocity of accelerated electrons gives access to the tension $V(t)$ of the emitting conductor [48, 49]. In (d), a circuit is analysed by a modified electronic microscope where the primary electron beam is delivered by picosecond lightning of a photocathode [50].] 
être injectée par une impulsion lumineuse, ce qui permet l'émulation sélective d'une partie quelconque de ce circuit.

La génération d'impulsions électriques en propagation libre a aussi été obtenue, d'une part en couplant un interrupteur photoconducteur à une antenne micro-onde [35-38], et d'autre part en utilisant le redressement optique [39] (cet effet non linéaire du deuxième ordre génère une polarisation électrique : $P(t)=\chi_{0}^{(2)} E(t)^{2}$ sous l'effet d'un champ électrique lumineux $E(t))$. La rapidité du redressement optique est limitée par l'absorption micro-onde des matériaux optiques à une fraction de picoseconde [30]. Ces techniques de génération d'impulsions électriques se propageant librement pourraient éventuellement permettre l'excitation d'un circuit électronique sans contact, bien que cette possibilité reste encore à explorer.

2. Sondage de tensions par laser (Fig. 3). - Un champ électrique $E_{0}(t)$ induit un changement de l'indice de réfraction $n$ dans un matériau électrooptique [40]:

$$
\Delta n(t)=\frac{2 \pi}{n^{2}} \chi^{2} E_{0}(t)
$$

Après une utilisation de cet effet, appelé aussi effet Pockels, pour obtenir des modulateurs électro-optiques, une application plus récente a été faite à la lecture de signaux électriques sur des lignes ou circuits électroniques [41-43].

Cette technique de sondage électro-optique est très souple : elle permet aussi bien une mesure du champ électrique dans le susbtrat portant le circuit électronique s'il est électro-optique (ce qui est le cas du GaAs), qu'une mesure externe par un cristal « sonde " recouvrant une grande partie du circuit [44] ou au contraire constituant une microsonde [45, 46]. Quelques exemples de géométrie sont donnés en figure 4. Cette technique est à la fois sensible et peu perturbatrice [47]. L'état des possibilités actuelles permet une sensibilité de quelques dizaines de $\mu \mathrm{V} / \sqrt{\mathrm{Hz}}$ [48]. Une résolution temporelle subpicoseconde a été obtenue [28], limitée par les contraintes de propagation. Une résolution de $\sim 200$ fs a été obtenue en propagation libre [39].

Un interrupteur photoconducteur peut constituer un point test sur un circuit par échantillonnage du courant détourné vers ce point test [45]. Cette méthode de sondage par «switch » photoconducteur nécessite de poser des « switches » photoconducteurs
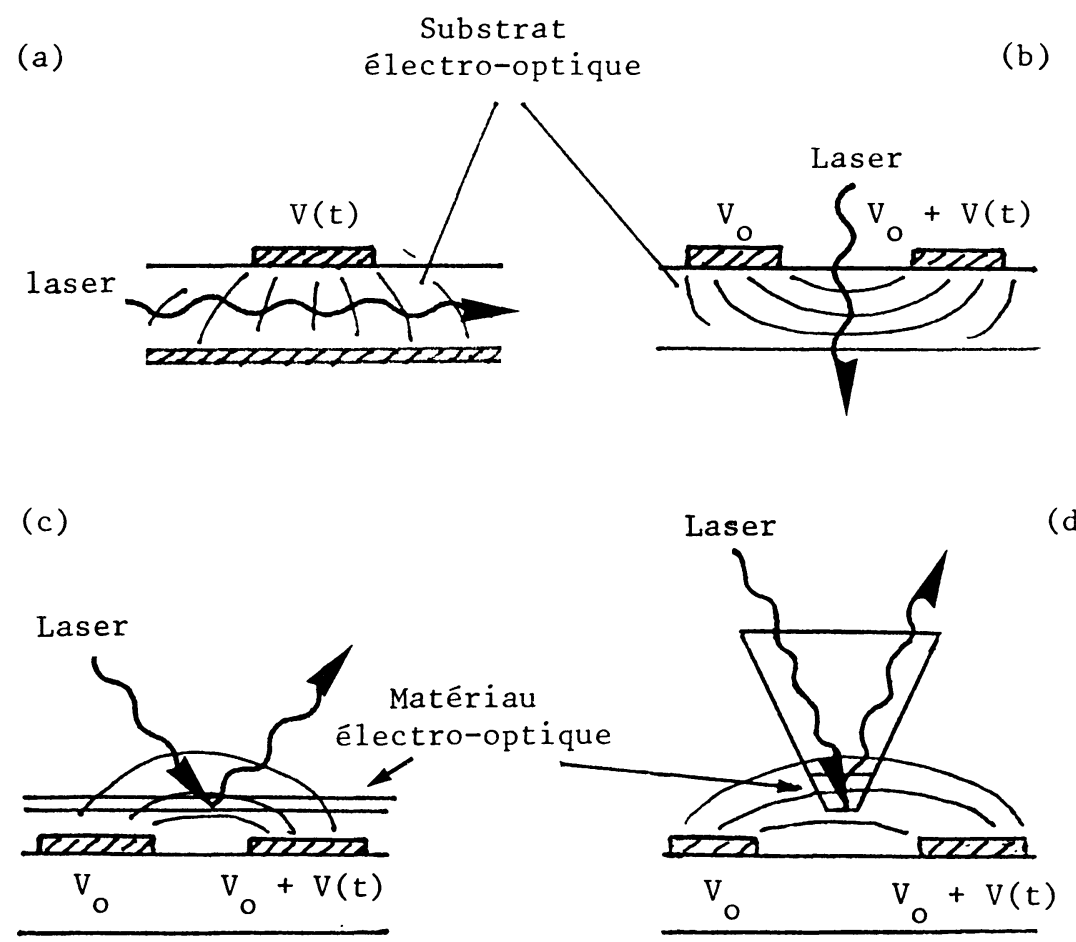

Fig. 4. - Sondage électro-optique d'une tension électrique. La biréfringence induite dans un matériau électro-optique peut être lue par une impulsion laser. Si le matériau portant le circuit est électro-optique, le signal de sondage est lu directement à travers le substrat $(\mathrm{a}, \mathrm{b})$. Un cristal de sonde électro-optique peut aussi être approché du circuit sur toute sa surface (c) ou en un point particulier (d).

[Electro-optic sampling of an electrical voltage. Birefringence induced in an electro-optic medium can be probed by a laser pulse. If the substrate of the circuit is electro-optic itself (as in the case of GaAs or InP), the probe signal can be read directly through it. A probe crystal can also be brought near the circuit on a large surface (c) or on a restricted area (electro-optic needle-d) [40, 41].] 
lors de l'élaboration du circuit. Le manque de souplesse de cette technique s'accompagne d'une perturbation du circuit par la mesure. Par contre, la résolution actuelle a été amenée à un régime subpicoseconde $[29,50]$. Une miniaturisation du procédé a été réalisée [51].

L'effet photoélectrique sur le conducteur transportant le signal électrique $V(t)$ permet l'émission d'électrons sous l'effet d'une impulsion laser. Une grille portée à un potentiel $V_{\mathrm{G}}$ permet d'accélérer les électrons émis. Leur énergie $W$ vaudra $q\left(V_{\mathrm{G}}-V(t)\right)$. L'analyse de cette énergie donne accès au potentiel du conducteur à l'instant de l'arrivée de l'impulsion lumineuse [52, 53]. Une résolution temporelle du sondage photoémissif de 5 ps a été obtenue. La sensibilité actuelle est encore médiocre $(10 \mathrm{mV} / \sqrt{\mathrm{Hz}})$. La contrainte apportée par cette méthode est de nécessiter un fonctionnement sous vide. Les circuits à tester ne doivent pas non plus être sensibles aux forts champs électriques nécessaires à l'accélération des électrons photoémis.

$\mathrm{Si}$ le faisceau d'électrons d'un microscope électronique à balayage est produit par effet photoélectrique, le contraste de potentiel d'un circuit peut être directement analysé par imagerie électronique [54]. La résolution temporelle atteinte (5 ps) et la sensibilité $(3 \mathrm{mV} / \sqrt{\mathrm{Hz}})$ du sondage par microscope électronique sont comparables à la technique précédente. Cette technique est assez lourde puisqu'elle nécessite un microscope électronique modifié, mais l'imagerie électronique permet une résolution spatiale $(0,2 \mu \mathrm{m}$ démontré) compatible avec une éventuelle intégration des circuits électroniques rapides.

3. Etude par laser d'un circuit électronique. - Le test du circuit électronique demande à la fois l'excitation du circuit par une impulsion électrique, puis le sondage du signal électrique émis. En utilisant les
EXCITATIONDU CIRCUIT

Générateur électrique
d'impulsions récurrentes Générateur électrique
sinusoiddal

Switch photoconducteur

Photodiode

Phototransistor

Redressement optique

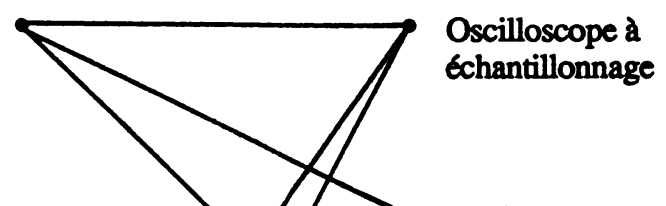

Analyseur de spectre ou de réseau

Sondage électro-optique

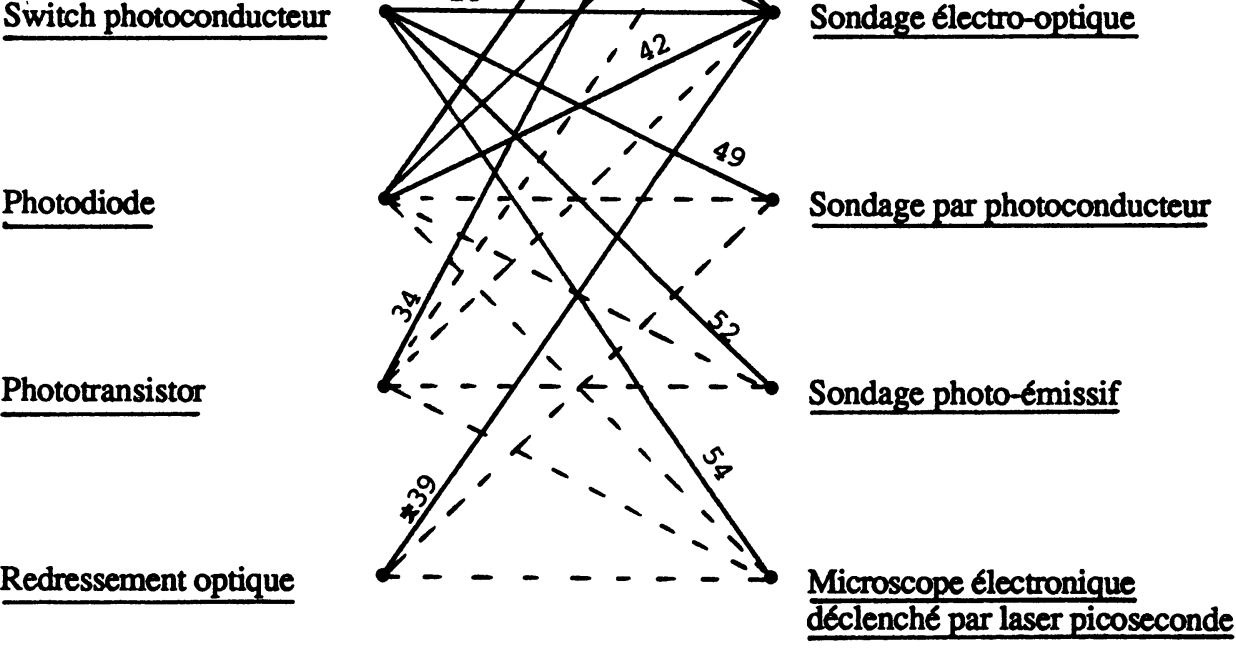

démontré

........ non démontré

Fig. 5. - Schéma illustrant différentes combinaisons de techniques d'excitation de circuit et d'analyse du signal de sortie, démontrées (traits pleins avec référence) ou non encore démontrées (tirets). A côté des techniques classiques, un grand nombre des possibilités sont offertes par l'utilisation des lasers picosecondes (soulignées). L'astérisque indique une démonstration sur un signal électrique en propagation libre, donc sans application possible à un circuit.

[Illustration of different combinations for excitation and probing of an electronic circuit, demonstrated (full lines with reference) or not (dotted lines). Beside classical technics, large possibilities are offered by the use of picosecond lasers (underlined). The star indicate a demonstration to free propagation, and thus without application to a circuit.] 
techniques décrites précédemment, l'échantillonnage de la réponse du circuit peut être obtenu entièrement par des moyens optiques. Mais un circuit peut aussi être étudié par une méthode hybride où seule l'excitation ou la détection sont effectuées optiquement.

Le schéma de la figure 5 donne un large éventail des possibilités offertes, démontrées ou non encore mises en œuvre.

Ainsi, à côté des techniques de test purement électronique (excitation du circuit par des impulsions électriques, ou par un signal hyperfréquence sinusoïdal, suivie d'une détection par un oscilloscope à échantillonnage ou par un analyseur de spectre ou de réseau), se développent des techniques d'analyse de circuits électroniques rapides par des techniques optiques. Une technique purement optique, telle que l'excitation par «switch » photoconducteur suivie du sondage électro-optique [28], est la plus performante : l'excitation ultra-rapide du circuit permet d'en obtenir la réponse impulsionnelle en régime linéaire ou non linéaire, tandis que le sondage électro-optique permet l'analyse du circuit point par point sans contact et sans nécessiter de réaliser une quelconque adaptation d'impédance. Une technique hybride, où seul le sondage est effectué optiquement par effet électro-optique [48, 57], met à profit l'analyse sans contact du circuit tout en conservant la simplicité de l'excitation hyperfréquence. Une autre technique hybride souple est celle utilisant l'excitation optique des transistors du circuit (qui sont tous des phototransistors) par un laser picoseconde, tandis que la réponse du circuit est analysée par un oscilloscope à échantillonnage.

Ces techniques optiques ont déjà été appliquées à des études de transistors rapides. A côté de la technique électronique de mesure de la fréquence de résonance d'une boucle constituée de transistors identiques ou de la technique utilisant un analyseur de réseau [58], les possibilités d'étude d'un transistor unique sont illustrées par la figure 6 (issue des Réf. $[59,60])$. A noter la réponse négative du transistor aux temps courts due à l'émission d'antenne de la base excitée et qui montre une limitation de la résolution spatiale inhérente aux circuits hyperfréquences.

\section{Exemple de l'étude de composants optoélectroni- ques.}

a) Etude d'un photodétecteur. - L'analyse de la réponse impulsionnelle d'un détecteur rapide peut être effectuée en excitant le détecteur par une impulsion lumineuse brève et en analysant le signal électrique délivré par l'une des techniques décrites précédemment (Fig. 7). Si le temps de réponse du détecteur est plus lent que 20-25 ps, un oscilloscope à échantillonnage est un moyen de détection suffi- sant. Le traitement du signal électrique par un analyseur de spectre permet d'étendre cette mesure à de plus hautes fréquences, mais l'information sur les phases est perdue. Par cette technique, ont été mesurées les bandes passantes de détecteurs jusqu'à $40 \mathrm{GHz}$ ( $\sim 5 \mathrm{ps})$ [55]. L'utilisation de lasers à semiconducteurs à injection [61] limite encore la résolution temporelle par rapport à l'utilisation d'un laser à modes couplés picoseconde ou femtoseconde. Le sondage électro-optique du signal électrique émis donne de façon purement optique la fonction réponse du détecteur [42]. C'est la seule technique simple permettant de déterminer complètement une réponse plus rapide que $20-25$ ps.

A noter que, si l'on cherche seulement à obtenir la bande passante du détecteur, l'utilisation d'impulsions lumineuses brèves n'est pas nécessaire. Une excitation optique sinusoïdale suivie d'une analyse de spectre du signal électrique permet d'obtenir cette information limitée. L'excitation optique modulée peut être obtenue par battement de deux lasers monofréquence $[62,63]$ ou par conversion phase-amplitude de l'émission optique d'un laser modulé en fréquence [64].

b) Analyse de l'émission lumineuse transitoire d'une diode laser. - Un outil très puissant pour l'analyse d'un émetteur lumineux à l'échelle picoseconde est la caméra à balayage de fente (« Streak camera »). Une résolution de quelques picosecondes peut être atteinte, limitée le plus souvent en pratique à une dizaine de picosecondes par le « jitter » des circuits de synchronisation. Un matériel commercial, d'utilisation aussi simple qu'un oscilloscope (mais à entrée optique), est depuis peu disponible (Hamamatsu 00S-01 (10 ps)). La puissance de cet outil est néanmoins limitée à la détection du spectre visible par l'utilisation de photocathodes à effet photoélectrique.

L'utilisation d'une caméra à balayage de fente associée à une source laser picoseconde donne un moyen très souple d'étude de la dynamique d'une émission lumineuse. En dehors du domaine d'utilisation de la caméra à balayage de fente (résolution temporelle ou spectrale insuffisante), l'échantillonnage optique de l'émission lumineuse est possible par somme de fréquences avec une impulsion lumineuse brève qui constitue une porte optique ultrarapide [65]. Les différentes possibilités d'étude d'une émission optique transitoire vont être illustrées par l'étude de l'émission d'une diode laser :

Classiquement, une impulsion électrique « carrée » est ajoutée à la polarisation continue du laser, tandis que l'émission est détectée par une photodiode rapide et un oscilloscope à échantillonnage. La résolution temporelle est limitée d'une part par le générateur électrique (à une cinquantaine de picosecondes de temps de montée) et d'autre part par l'oscilloscope à échantillonnage (25 ps actuelle- 

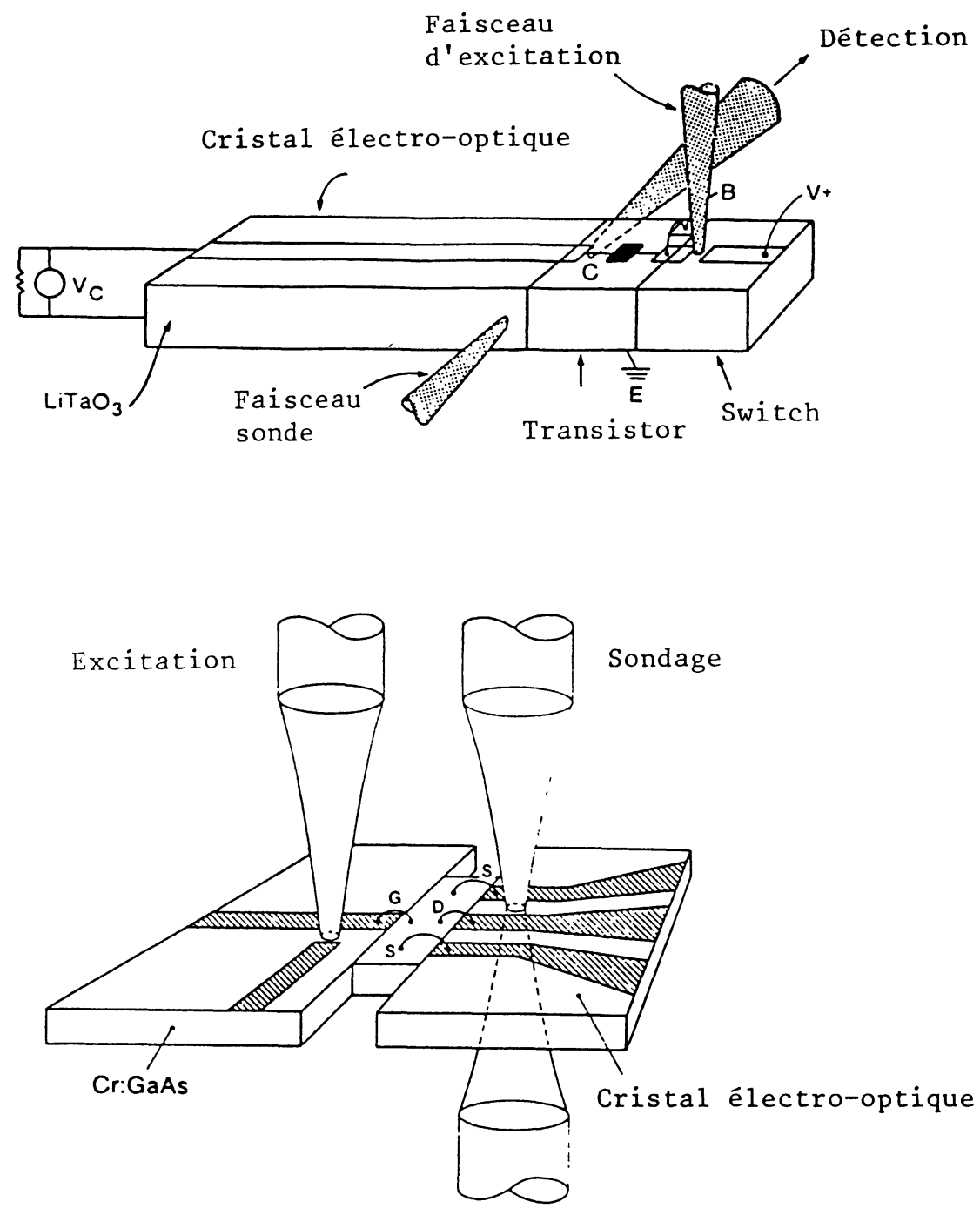

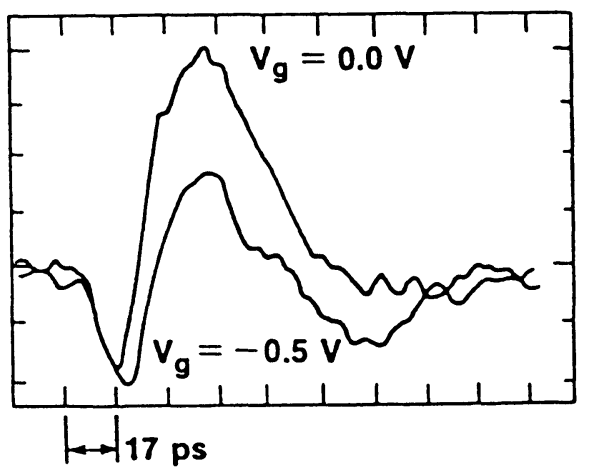

Réponse d'un transistor FET

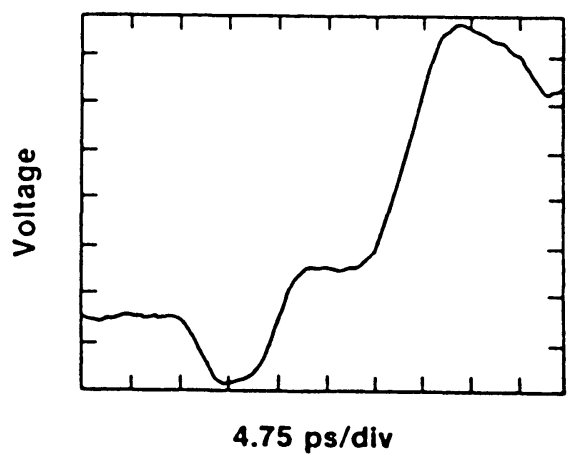

Réponse d'un transistor à base perméable

Fig. 6. - Deux exemples d'analyse d'un transistor rapide par l'usage de techniques laser. Dans ces deux exemples tirés des références [59] et [60], l'excitation de la base du transistor se fait par un «switch » photoconducteur, l'analyse du signal de sortie par effet électro-optique.

[Two examples of analysis of an ultrafast transistor by picosecond laser techniques, from references [59, 60]. The excitation of the transistor is derived from a photoconductive switch, while the output signal is analysed by electro-optic sampling.] 
Laser picoseconde

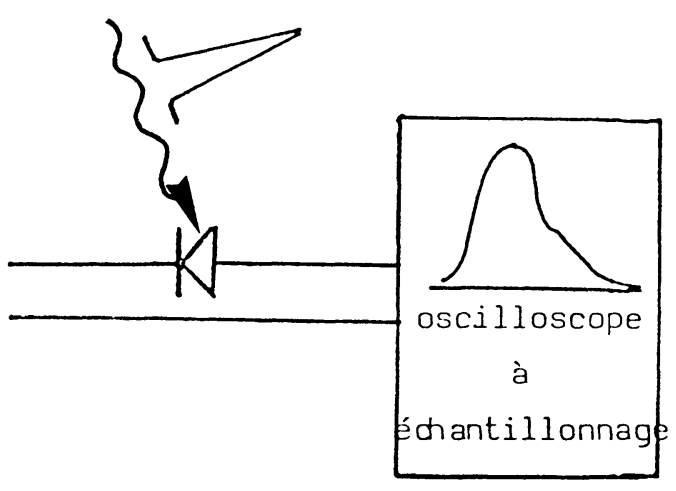

Echantillonnage électronique

(a)

Laser picoseconde

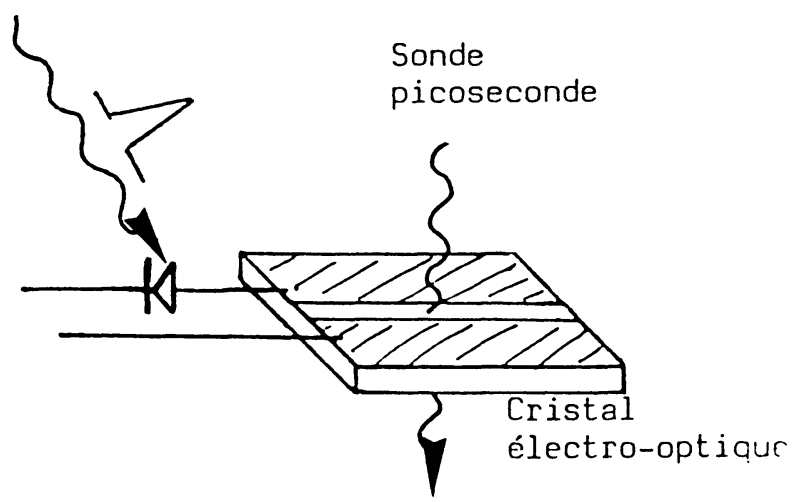

Sondage électro-optique

(c)

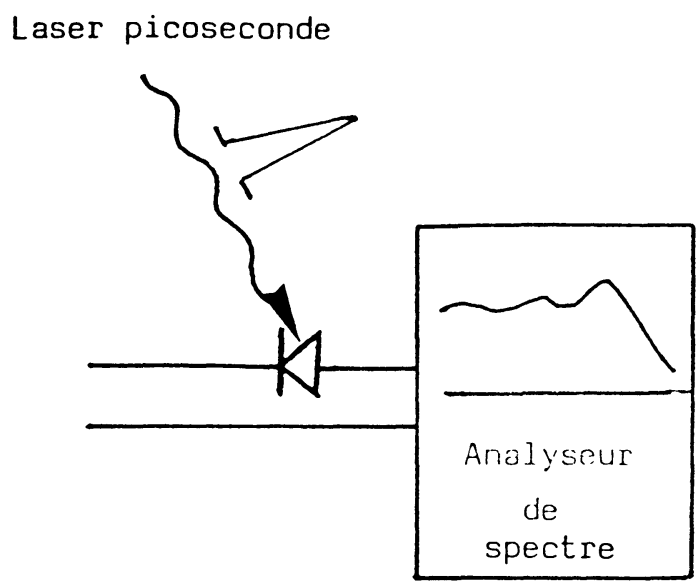

Analyse de spectre

(b)

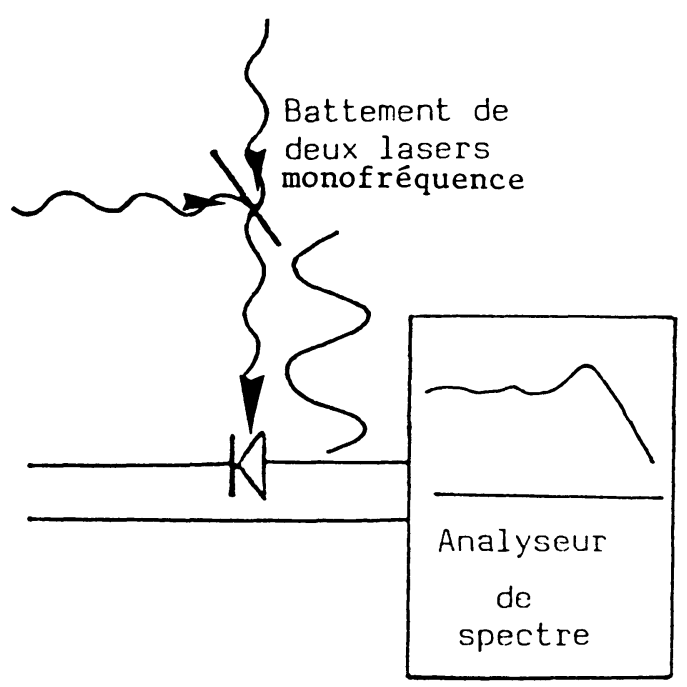

Echantillonnage spectral

(d)

Fig. 7. - Possibilité d'étudier la réponse d'un photodétecteur rapide. Après éclairement du photodétecteur par une impulsion laser picoseconde, le signal de sortie peut être analysé par un oscilloscope à échantillonnage (a), un analyseur de spectre (b) ou par sondage électro-optique (c). L'impulsion lumineuse brève peut être remplacée par une excitation sinusoïdale (obtenue par battement de deux lasers continus) suivie d'une analyse de spectre (d).

[Analysis of the response of an ultrafast photodetector. After lightning the photodetector by a picosecond laser pulse, the output signal can be analysed by a sampling scope (a), a spectrum analyser (b) or by electro-optic sampling (c). The ultrafast light pulse can be alternatively replaced by a sinusoidal excitation (derived from the beating of two continuous lasers) followed by a spectrum analysis (d).]

ment). La mesure nécessite d'autre part l'utilisation d'une photodiode rapide bien caractérisée afin d'être assuré que l'on analyse la dynamique du laser à l'aide de la photodiode (et non l'inverse!). La détection peut être améliorée en utilisant une caméra à balayage de fentes, amélioration limitée aux lasers émettant dans le visible (à GaAs par exemple), mais plus difficile pour les lasers émettant à 1,3 ou $1,5 \mu \mathrm{m}$.

Un «switch» photoconducteur placé sur la ligne de polarisation permet de générer des impulsions électriques excitatrices de faible durée (ou à front de montée rapide). Des durées de 17 ps ont été utilisées pour exciter des lasers à In-Ga-As-P [66]. La détection par une caméra à balayage de fentes est possible [67] en étendant la sensibilité dans l'infrarouge par doublement de fréquence [68]. L'amélioration des oscilloscopes à échantillonnage permettra certainement d'atteindre prochainement une résolution de 10 ps plus simplement. Les variations à partir de ce 
schéma d'excitation sont nombreuses : il est possible d'exciter optiquement le laser [69], ou d'utiliser une injection sinusoïdale $[62,67]$. On peut aussi envisager d'utiliser une photodiode rapide pour générer l'impulsion électrique.

Excitation et détection peuvent être effectuées à l'aide du même laser picoseconde : l'impulsion électrique est déclenchée par l'illumination d'un «switch» photoconducteur. L'échantillonnage de l'émission laser est obtenu par somme de fréquence avec une impulsion du même laser picoseconde retardé $[66,70]$. La résolution temporelle n'est plus limitée que par la durée des impulsions lumineuses, aussi bien pour l'excitation que pour la détection.

Un dernier exemple d'étude de composant optoélectronique est apporté par la caractérisation récente d'un modulateur optique de $100 \mathrm{GHz}$ de bande passante [71]. La configuration expérimentale est très voisine de celles permettant l'étude de l'émission optique d'un laser modulé directement.

En conclusion, comme le montrent des exemples d'analyse de composants discrets, les techniques basées sur l'utilisation des lasers picosecondes permettent maintenant l'analyse de la dynamique de circuits optoélectroniques ultra-rapides. La combinaison des techniques disponibles permet d'envisager l'étude d'un circuit optoélectronique quelconque en bloc ou élément par élément. On peut ainsi envisager la possibilité d'étudier les caractéristiques d'un détecteur associé à son amplificateur ou d'une diode laser associée à son circuit de commande.

\section{Perspectives et applications A des mesures} OU DIAGNOSTICS OPTIQUES. - Par rapport aux techniques classiques, un avantage important du sondage électro-optique vient de la possibilité d'étudier tout point d'un circuit sans contact, donc sans adaptation d'impédance et sans limitation par les connecteurs. Les limites actuelles de ces techniques viennent de la difficulté d'obtenir une excitation sans contact du circuit sauf par excitation des phototransistors du circuit lui-même, ce qui manque de généralité. Les possibilités d'excitation électrooptique d'un circuit par un cristal proche ou par le rayonnement d'une micro-antenne excitée par laser pourraient lever cette difficulté.

L'avènement industriel des techniques de test sans contact de circuits électroniques est proche. Des systèmes pré-industriels existent déjà (EGG-PAR). La diffusion de ces techniques dans les laboratoires industriels sera limitée tant que des systèmes de mesure compacts ne pourront être conçus. Les sources lasers utilisées pour les systèmes prototypes actuels sont assez complexes. Une voie nouvelle est ouverte par l'utilisation possible des lasers à semiconducteurs [45, 72-74]. Le développement de lasers picosecondes à semi-conducteurs affirmera certainement la viabilité industrielle des techniques laser de test électronique, et permettra de supporter la concurrence de nouvelles méthodes comme le microscope électronique à balayage stroboscopé [20, 21], ou l'analyse de l'émission de micro-antennes.

Par ailleurs, le développement de sources picosecondes simples permettrait une éclosion à beaucoup plus grande échelle d'appareils les intégrant. Quelques possibilités sont illustrées dans la suite.

1. L'oscilloscope picoseconde. - L'intégration d'un système de mesure électronique ne pose pas d'obstacle de principe : le développement de sources picosecondes simples provoquera immédiatement la généralisation des oscilloscopes ultra-rapides à échantillonnage basés soit sur le sondage électro-optique, soit sur un switch photoconducteur remplaçant les diodes tunnel des têtes à échantillonnage [75].

On peut aussi concevoir un oscilloscope électrooptique permettant la visualisation du signal électrique en temps réel (sans échantillonnage) en sondant le dispositif électro-optique à l'aide d'une succession d'impulsions lumineuses que l'on peut démultiplexer si elles ont des fréquences différentes. Bien qu'une démonstration ait déjà été effectuée [76] en utilisant une impulsion «chirpée » par dispersion et malgré son intérêt certain, cette technique semble délicate pour avoir un avenir à court terme.

2. Générateur électrique ultra-rapide. - Par la mise en œuvre d'un «switch » photoconducteur ou un photodétecteur ultra-rapide éclairés par un laser picoseconde, il est possible de générer des impulsions électriques très brèves (quelques ps) en utilisant un photoconducteur dopé pour raccourcir la durée de vie des porteurs. Il est possible d'obtenir des impulsions carrées ayant un temps de montée aussi court en utilisant un photoconducteur non dopé tel que ceux déjà utilisés pour synchroniser les caméras à balayage de fentes [33].

3. Caractérisation de dispositifs en optique guidée. La mesure de temps de retour d'une impulsion lumineuse rétroréfléchie dans un dispositif (fibre, modulateur) permet de localiser des imperfections du guidage. La détection par un oscilloscope à l'échantillonnage (commercialisée par Opto-Electronics, Ontario, USA) pourrait être améliorée en utilisant une détection optique, interférométrique ou par somme de fréquences. La mesure des caractéristiques optiques du milieu traversé, telle que la dispersion chromatique, est aussi possible [77].

4. Fluorimètre picoseconde. - La mesure des durées de vie de fluorescence nécessite le plus souvent une grande sensibilité. Les portes optiques permettant ce type de mesure sont utilisées de façon courante en laboratoire : la porte Kerr [78] demande une forte puissance laser, la caméra à balayage de fentes ou l'up-conversion de fluorescence [79] sont des techniques très performantes, tandis que l'amplification 
paramétrique est une technique qui peut se révéler satisfaisante dans certains cas [80].

La mesure des temps de vie de fluorescence peut être un test critique de la qualité de matériaux semiconducteurs. Actuellement, ce type de mesures est effectué en laboratoire [81]. L'analyse des impuretés est effectuée à plus grande échelle par analyse en fréquence de la photoluminescence. Le développement de fluorimètres picosecondes simples d'emploi et basés sur les techniques exposées plus haut donnerait une possibilité d'analyse complémentaire.

\section{Capteurs optiques utilisant un laser picoseconde.}

- Un grand nombre de capteurs optiques ont vu le jour surtout depuis la disponibilité de fibres optiques et de lasers à semi-conducteurs de bonne qualité et de faible coût. La précision de certaines mesures serait améliorée par l'emploi de lasers à impulsions brèves.

L'évaluation d'une distance par mesure de temps de vol est une technique de télémétrie optique. La précision de la mesure étant limitée par la durée des impulsions lumineuses, l'utilisation d'un laser picoseconde associé à une porte optique a été proposée très tôt pour améliorer la précision de la mesure [82, 83]. La mise en évidence de défauts dans un dispositif en optique guidée cité ci-dessus en est un exemple. Une extension dans le domaine femtoseconde a été récemment démontrée [84]. Une autre technique de télémétrie laser utilise la modulation du faisceau laser et mesure la différence de phase du signal renvoyé par l'objet par rapport à cette référence [85]. L'utilisation de lasers à modes couplés correspondrait à une modulation à une harmonique élevée de la cadence de répétition des impulsions. Un gain sur la précision de mesure de plusieurs ordres de grandeur pourrait être obtenu par rapport aux techniques de modulation classiques sans apporter de pertes. On pourrait ainsi faire la jonction avec les techniques interférométriques.

Le gyroscope laser utilise l'effet Sagnac pour différencier les trajets optiques des deux faisceaux se propageant en sens inverse dans un interféromètre en rotation [86]. Dans un gyrolaser, les deux fréquences émises en sens inverse sont différentes. Leur écart est proportionnel à la vitesse de rotation, sauf aux faibles vitesses où les deux fréquences se «bloquent » dans la zone aveugle [87], à cause de rétrodiffusions dans la cavité qui couplent les deux ondes. Plusieurs méthodes permettent de limiter cet effet $[88,89]$. Une autre technique a été proposée et sommairement testée [90] : si le gyrolaser est à modes couplés et si les impulsions ne se croisent dans aucun des éléments optiques de la cavité, l'influence des rétrodiffusions sera considérablement diminuée par la non-simultanéité des ondes ainsi superposées (Fig. 8). Les mêmes améliorations peuvent être apportées dans le cas d'un gyroscope à
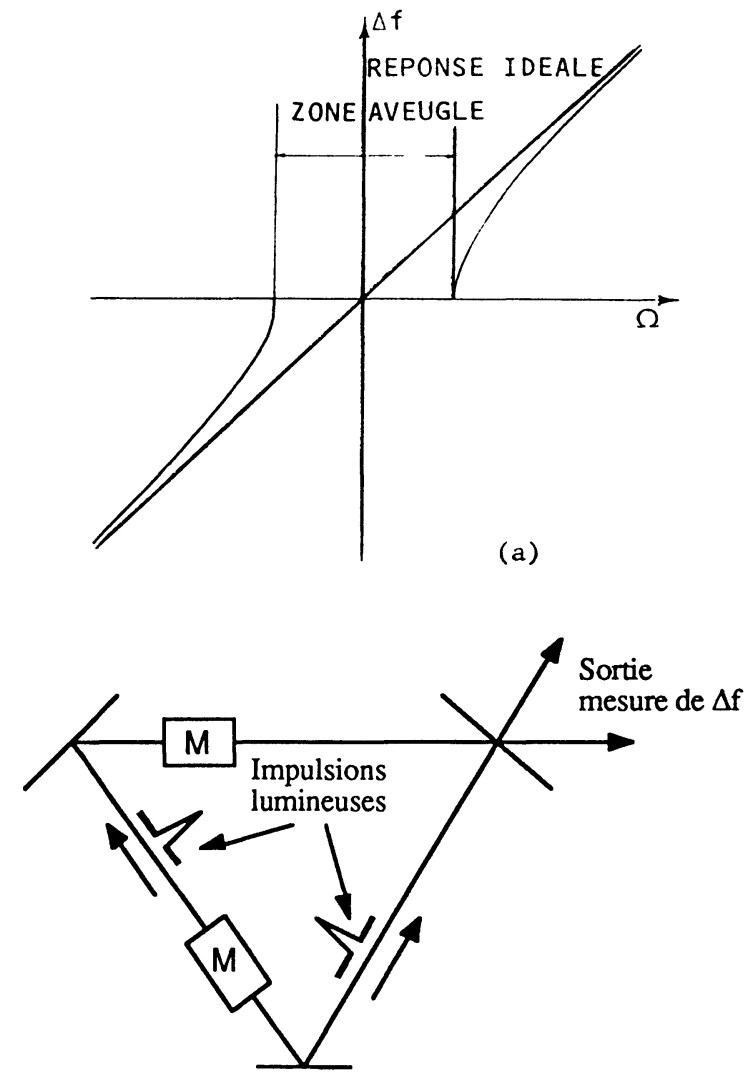

(b)

Fig. 8. - Dans un gyrolaser actif, la mesure des basses vitesses de rotation donnée par le battement des deux fréquences se propageant en sens inverse n'est plus pratiquable (a) à cause du couplage des deux ondes par les rétrodiffusions des éléments diffusants de la cavité. Si ces deux ondes sont maintenant des impulsions brèves (b) obtenues par couplage de modes (à l'aide des modulateurs $\mathrm{M})$, le couplage est amoindri si elles ne se croisent pas dans les éléments diffusants de la cavité.

[In an active gyrolaser, the measurement of low rotation rates is no more practicable (a) due to the coupling of the two counterpropagating waves by backscatting on the optical elements of the cavity. If the two waves are now ultrashort pulses (b) obtained by mode locking (with the modulators $\mathrm{M}$ ), the locking effect of backscattering is reduced if the two pulses do not cross in scattering elements.]

cavité externe. Cette application présente la particularité de ne pas utiliser la résolution temporelle des impulsions picosecondes, mais leur limitation dans l'espace [91].

\section{Applications aux télécommunications optiques.}

Les progrès récents des télécommunications optiques permettent d'atteindre des cadences de répétition supérieures au Gbit/s commercialement et supérieures à $10 \mathrm{Gbits} / \mathrm{s}$ en laboratoire. Parmi les nombreuses possibilités permettant d'augmenter le débit des 
communications optiques (multiplexage, détection cohérente, ...), l'utilisation des lasers à modes couplés a été proposée. Les possibilités seront analysées dans la suite.

Un autre secteur en développement est constitué par les communications hyperfréquences où certains composants HF sont remplacés par des éléments optiques, en particulier pour le transport des signaux. L'application des lasers à modes couplés à ce domaine est une activité en pleine expansion.

A. Les lasers À MOdes COUPLÉS POUR LES TÉlÉCOMMUNICATIONS MULTIGIGABITS. - Les composants optoélectroniques atteignent des bandes passantes très élevées. Des détecteurs répondant à plusieurs dizaines de $\mathrm{GHz}$ ont été démontrés [55]. Des lasers à semi-conducteurs peuvent être modulés à forte cadence aussi $(26,5 \mathrm{GHz})$ [92]. Les modulateurs électro-optiques atteignent aussi plusieurs dizaines de $\mathrm{GHz}$ de bande passante [71, 102-104], même si les composants sur GaAs, les plus rapides, ont perdu de l'intérêt pour les télécommunications.

L'électronique hyperfréquence progresse rapidement: les transistors bipolaires sur silicium atteignent $\sim 3 \mathrm{GHz}$, tandis que les semi-conducteurs III$\mathrm{V}$ devraient permettre de concevoir des circuits dans la bande $30-50 \mathrm{GHz}([96,97]$ en plus des références déjà citées). La bande passante des fibres optiques est elle aussi très grande : la limite théorique de propagation est de $\sim 1000 \mathrm{~km}$ à $10 \mathrm{Gbits} / \mathrm{s}$ pour une dispersion de $2 \mathrm{ps} / \mathrm{nm} . \mathrm{km}$ (mais tomberait néanmoins à $10 \mathrm{~km}$ à $100 \mathrm{Gbits} / \mathrm{s})$, et la modification de la dispersion des fibres permettrait encore d'améliorer ces performances [98].

Ainsi, les télécommunications multigigabits semblent disposer de tous les éléments pour se développer en laboratoire au-delà des possibilités actuelles $[99,100]$ mais en utilisant simultanément des composants très sophistiqués.

1. Multiplexage-démultiplexage à l'aide de lasers à modes couplés. - L'électronique est actuellement le maillon le plus faible d'un système de télécommunications à haut flux. Une démonstration d'utilisation des lasers à modes couplés a été faite permettant d'utiliser une électronique nettement plus lente que le taux de transmission de l'information (8 Gbits/s manipulé par une électronique à $2,6 \mathrm{GHz}$ ) [101].

Le schéma en est donné figure 9 : un laser à modes couplés délivrant des impulsions de 15 ps à la cadence de $4 \mathrm{GHz}$ constitue l'horloge du système. Il est divisé en deux voies encodées séparément par deux modulateurs et qui sont ensuite multiplexées à $8 \mathrm{GHz}$. A la réception, après démultiplexage, les deux canaux à $4 \mathrm{GHz}$ sont décodés séparément. L'amélioration du multiplexage et du démultiplexage

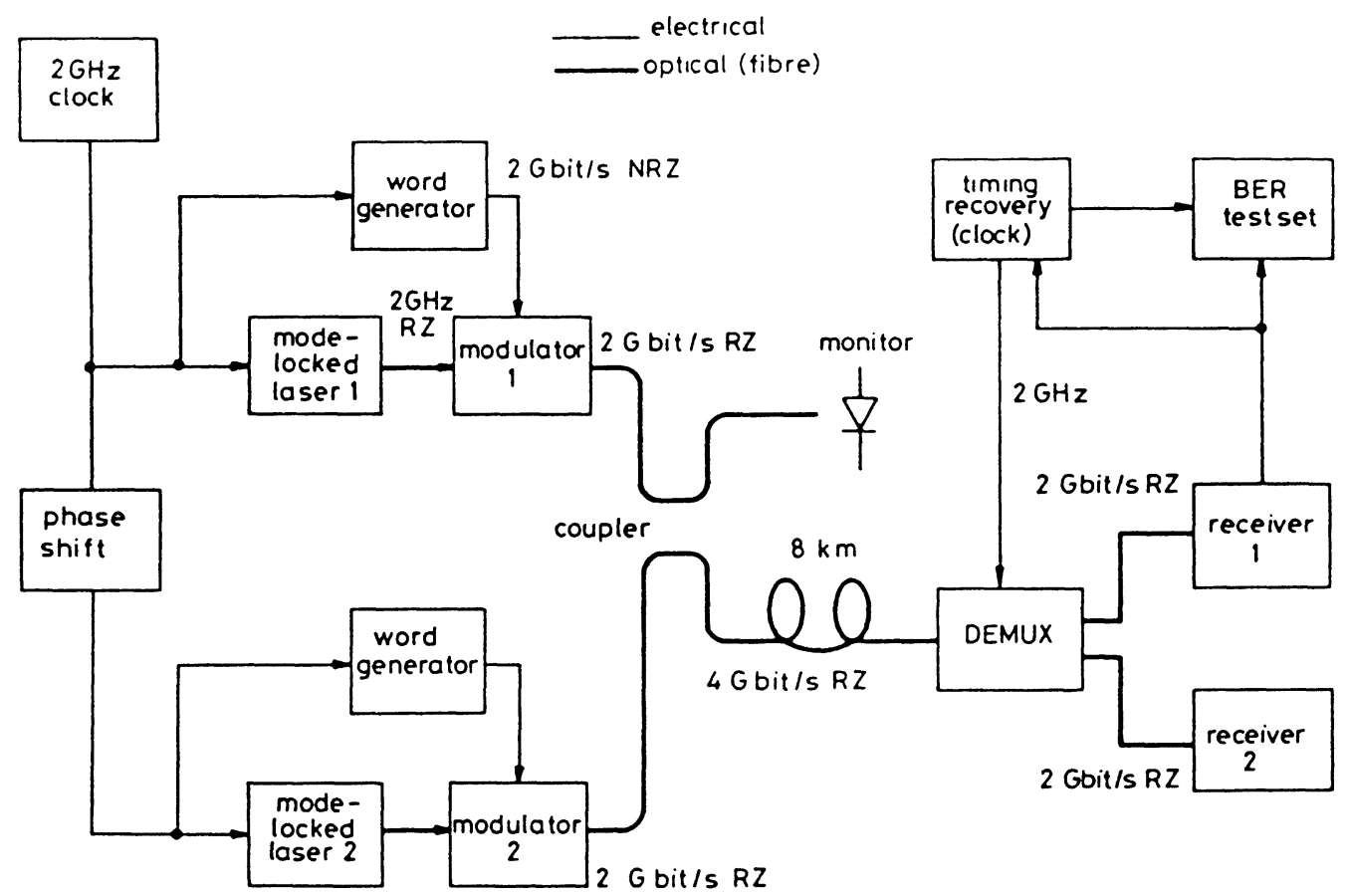

Fig. 9. - Multiplexage-démultiplexage par lasers à modes couplés. Le train d'impulsions optiques délivrées par un laser à modes couplés constitue l'horloge et le support de l'information [101, 102]. Après encodage par un modulateur, les signaux sont multiplexés par un coupleur. A la détection, les signaux sont démultiplexés par un modulateur.

[Time division multiplexing-demultiplexing using mode-locked lasers. The optical pulse train delivered by a picosecond mode-locked diode laser constitutes the clock and is encoded by the information [101, 102]. After encoding by a modulator, signals are multiplexed by a coupler. At the output of the fibre, the signals are demultiplexed by a modulator.] 
a permis d'atteindre la cadence de 16 Gbits/s [102], ce qui fait de cette technique la plus performante actuellement. Le multiplexage à $100 \mathrm{Gbits} / \mathrm{s}$ d'impulsions lasers de 6 ps obtenues par commutation du gain d'un laser à semi-conducteurs a par ailleurs été démontré [103] (le démultiplexage reste néanmoins problématique).

La largeur spectrale des impulsions picosecondes peut sembler un handicap pour la distance de propagation permise dans une fibre. En fait, si l'impulsion lumineuse dure un tiers du temps entre impulsions, ce qui est suffisant pour appliquer la technique décrite, l'étalement spectral est voisin de celui obtenu en régime continu avec une modulation ayant une bande passante égale à trois fois la fréquence d'horloge. La difficulté actuelle liée à cette technique vient de l'utilisation d'un laser à modes couplés à cavité externe [104]. Pour évaluer l'avenir de cette technique, il faut aussi prendre en compte la concurrence des techniques de multiplexage en fréquence qui présentent des avantages potentiels [105].

2. Transmission dans le mode soliton. - Le débit d'information se propageant sur une fibre est limité de façon ultime par la dispersion chromatique. Dans certaines conditions (intensité suffisante, dispersion «négative »), la dispersion résiduelle peut être compensée par un effet non linéaire, l'effet Kerr optique, donnant une impulsion qui se propage indéfiniment sans déformation : le soliton [106-108]. Il a été proposé que ces conditions soient la solution aux problèmes des télécommunications à longue distance et haut débit [109].

Cette proposition se heurte à certaines difficultés. L'absorption résiduelle de la fibre doit être compensée périodiquement, faute de quoi le soliton s'étale temporellement $[110,111]$. D'autre part, l'interaction entre solitons successifs ne peut être négligée. Un espacement entre impulsions de plusieurs dizaines de fois leur durée serait nécessaire pour éviter les collisions de solitons [112]. Enfin, il est nécessaire de disposer d'impulsions lumineuses intenses $(\sim 1 \mathrm{~W})$ non encore disponibles simplement et pour lesquelles les autres effets non linéaires, comme l'effet Raman stimulé, ne peuvent être négligés et amènent une instabilité en fréquence du soliton.

Les solitons optiques donnent lieu à des phénomènes physiques fascinants et seront certainement longtemps encore l'objet de recherches fondamentales. Comme solution aux problèmes de télécommunications à longue distance, la propagation dans le mode soliton progresse rapidement en laboratoire [113]. Mais, raisonnablement, pendant de nombreuses années, la propagation optique linéaire dans des fibres à dispersion modifiée répondra plus simplement aux problèmes de propagation sans déformation à longue distance.
B. LES APPLICATIONS AUX HYPERFRÉQUENCES. La manipulation de signaux hyperfréquences est l'objet de nombreux progrès, d'abord par l'utilisation des semi-conducteurs à réponse rapide, diodes et transistors $[114,115]$ et grâce au développement des techniques de commande optique [116]. Les perspectives d'utilisation sont ainsi multipliées, en allant de la miniaturisation des accès à des systèmes radars permettant la mise en œuvre d'antennes adaptatives [117] jusqu'à la simplification des moyens de communications hyperfréquences [118].

1. Manipulation optique des composants. - Un plasma photo-induit dans un semi-conducteur placé dans un guide d'onde permet d'en moduler les pertes. Des atténuations importantes ont été obtenues jusqu'à $40 \mathrm{GHz}[119,120]$. Des déphasages importants $\left(300^{\circ} / \mathrm{cm}\right)$ peuvent aussi être obtenus par cette méthode [121].

Des « switches » photoconducteurs placés sur des lignes coplanaires permettent d'obtenir des interrupteurs ultra-rapides (voir chapitres précédents). L'interruption optique de cavité RF permet de générer des impulsions hyperfréquences brèves [122].

L'excitation quasi continue de cavités hyperfréquences a aussi été démontrée [123, 124]. Un tel "switch" photoconducteur peut être considéré comme un mélangeur ayant une sortie électrique, mais deux entrées, l'une électrique, l'autre optique. L'oscillateur local peut être considéré comme optique, dans ce cas, un signal électrique est contrôlé optiquement [125]; ou bien l'oscillateur local est électrique [126], le composant constituant alors une porte optique. Le couplage d'un «switch" photoconducteur à une antenne d'émission a été aussi envisagé dans l'espace [35], dans le plan [30-32] ou dans une structure guidée [127]. L'accès optique à des diodes PIN donne d'importantes possibilités de moduler la résistance et la capacité d'un composant hyperfréquence. Les commandes optiques de déphaseurs, atténuateurs, limiteurs, modulateurs et interrupteurs HF ont été obtenues [116-118]. Des composants actifs peuvent aussi être contrôlés optiquement tels des transistors rapides MESFET [128]. La manipulation de tels composants à l'aide d'impulsions lumineuses brèves est l'objet de recherches très actives.

2. Signaux optiques hyperfréquences (Fig. 10). - La modulation d'intensité d'un faisceau lumineux à haute fréquence permet, en utilisant par exemple un photodétecteur rapide ou un photoconducteur, de reconstituer un signal électrique HF. Sous sa forme optique, ce signal hyperfréquence peut être manipulé optiquement, ce qui présente des possibilités nouvelles. L'utilisation d'une fibre optique peut remplacer le guide d'onde rigide, d'où un gain important de souplesse, de poids et de bande passante. De 


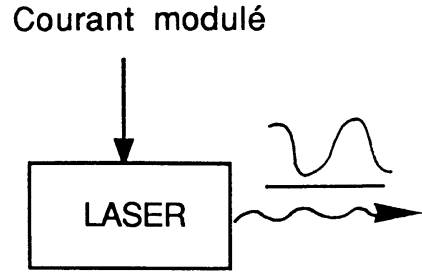

(a)

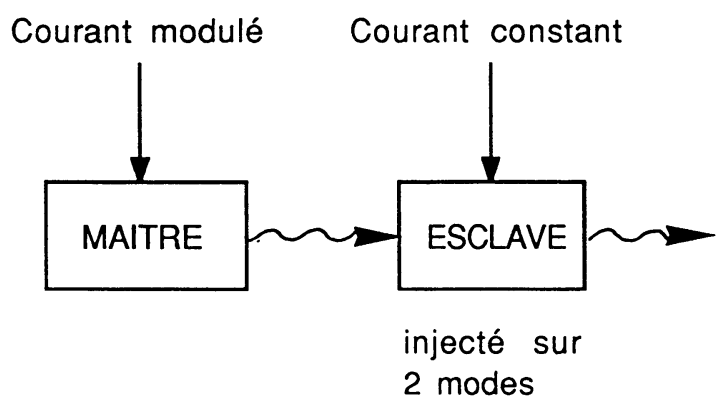

(c)

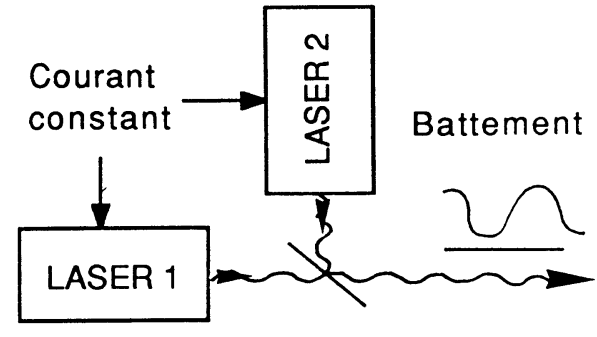

(b)
Courant modulé à la fréquence intermode

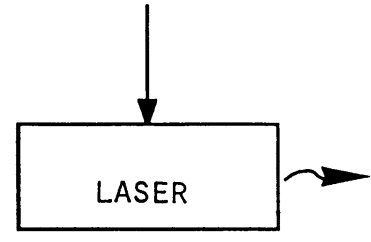

(d)

Fig. 10. - Différentes techniques de génération de signaux optiques modulés à très haute fréquence. La modulation directe d'un laser à semiconducteur (a) est limitée à environ $10 \mathrm{GHz}$. Le battement de deux lasers de fréquences différentes (b) passe outre cette limite. Le couplage des modes d'une cavité laser courte est une autre solution encore à peine explorée.

[Different techniques to generate optical signals modulated at high frequency. The direct modulation of a laser diode (a) is limited to around $10 \mathrm{GHz}$ (a). The beat of two single frequency lasers (b) do not present this limitation [134]. The mode coupling of a short laser cavity is an other solution hardly explored : two modes of a slave laser can be excited by a modulated master laser (c) [135], or the laser cavity can be directly modulated at the intermode frequency (d) [141].]

grandes distances peuvent être parcourues sans pertes. La miniaturisation associée permet d'envisager des antennes réseau ayant un grand nombre d'éléments [117, 129]. La fibre optique permet d'obtenir facilement de grands délais hyperfréquence ou des déphaseurs par enroulement sur un mandrin piézoélectrique ainsi que d'autres fonctions [130].

Les télécommunications optiques hyperfréquences apparaissent ainsi comme le prolongement des télécommunications optiques poussées à plus haute fréquence. Des démonstrations ont été effectuées à $10 \mathrm{GHz}$ en utilisant des diodes laser modulées directement ou par modulateur externe pour générer le signal optique HF [131]. Les composants actuels permettent d'atteindre une bande passante dépassant largement $20 \mathrm{GHz}$. Les pertes d'insertion de tels systèmes sont néanmoins importantes, malgré les précautions prises pour les adaptations d'impédance [132].

La modulation directe de lasers à semi-conducteurs à haute fréquence ouvre une possibilité très intéressante d'obtenir des signaux optiques hyperfréquences dans un domaine s'étendant jusqu'aux envi- rons de $20 \mathrm{GHz}$. Des problèmes importants sont encore posés outre cette limitation de la bande passante : la puissance électrique de commande est très supérieure à la puissance optique émise [131] et le signal optique est bruité. Le développement de lasers monomodes en régime dynamique est en voie de résoudre ces problèmes [133].

Une autre solution pour obtenir des signaux optiques hyperfréquences de fréquence plus élevée est de faire battre deux rayonnements monochromatiques de fréquence différente. Des démonstrations ont été réalisées en injectant par les bandes latérales d'un laser maître modulé deux lasers esclaves [134] ou deux modes d'un laser esclave [135]. Le signal optique modulé n'est plus limité vers les hautes fréquences ( $35 \mathrm{GHz}$ démontrés), mais cette possibilité se paie par la complexité du dispositif.

3. Les lasers à modes couplés comme source optique hyperfréquence. - Les lasers à modes couplés délivrent un train d'impulsions dont la période est déterminée par un aller et retour dans la cavité optique. Ce signal lumineux constitue ainsi une 
porteuse hyperfréquence de fréquence $\nu=\mathrm{c} / 2 \mathrm{nl}$, où $c$ est la vitesse de la lumière et $n$ l'indice de réfraction dans la cavité de longueur 1 .

Le couplage de modes des lasers à semi-conducteurs par injection de courant est mis en œuvre depuis le début des années 1980 et a fait l'objet d'un grand nombre de réalisations [136]. Des impulsions picosecondes de bonne qualité optique peuvent être obtenues [137, 138] et même des impulsions subpicosecondes par adjonction d'un absorbant saturable [139].

L'utilisation de cavités courtes (de longueur millimétrique), pour obtenir par couplage de modes une fréquence de récurrence dans le domaine des hyperfréquences, a été envisagée avec ou sans cavité externe [140-142] en recherchant un fonctionnement pour lequel la durée des impulsions lumineuses est voisine de leur fréquence de récurrence. Même si actuellement ces réalisations sont peu satisfaisantes à cause de la qualité optique limitée du train optique modulé et du contrôle insuffisant du composant, cette possibilité d'obtenir une émission optique modulée dans le domaine des hyperfréquences semble prometteuse.

\section{Conclusions.}

Dans cet article ont été envisagées les possibilités d'application des techniques de mesure en régime picoseconde ou femtoseconde. N'ont pas été considérés les domaines du traitement optique de l'information [143] pour lesquels des portes ultrarapides sont démontrées [144], ou encore de la commutation photonique ultrarapide [145], car ces domaines manquent encore de maturité.

Les applications industrielles les plus prometteuses pour les lasers à impulsions ultra-courtes concernent d'une part la mesure, en particulier sur des circuits électroniques, et d'autre part les applications optoélectroniques consistant en la manipulation optique de signaux électroniques.

Un développement de sources lasers picosecondes simples, de faible encombrement, permettrait non seulement d'obtenir des appareils de test optique utilisables hors des laboratoires spécialisés, mais apporterait aussi des possibilités d'applications dans le domaine de l'optoélectronique hyperfréquence.

Une réelle expansion de l'utilisation de ces techniques doit passer par la simplification des sources lasers actuelles lourdes (à YAG, colorants, centres colorés, ...). Là encore, comme dans d'autres domaines, les lasers à semi-conducteurs joueront un rôle irremplaçable, que l'obtention des impulsions brèves repose sur le couplage de modes ou sur la commutation du gain. On doit aussi considérer le rôle possible qui pourrait être joué par les lasers à solides pompés par diode laser.

\section{Remerciements.}

Je tiens à remercier les personnes des Laboratoires de Marcoussis qui m'ont aidé par d'utiles discussions, en particulier L. Torchin, M. Gaillard et J. M. Gabriagues.

\section{Bibliographie}

[1] Ultrashort Light Pulses, Ed. S. L. Shapiro (Springer Verlag, Berlin) 1977 ; Une nouvelle édition réactualisée : Ultrashort Light Pulses and Applications, Ed. W. Kaiser (Springer Verlag, Berlin) 1988.

[2] New G. H. C., Rep. Prog. Phys. 46 (1983) 877.

[3] Fork R. L., Brito-Cruz C. H., Becker P. C. and SHANK C. V., Opt. Lett. 12 (1987) 483.

[4] Ito H., Yokohama H., Maruta S. and Inaba H., Electron. Lett. 15 (1979) 738.

[5] Lin C., Liu P. L., DAMEN T. C., Eilenberger D. J. and HARTMAN R. L., Electron. Lett. 16 (1980) 600.

[6] Downey L. M., Bowers J. E., Tucker R. S. and Agyekum E., IEEE J. Quant. Elec. QE 23 (1987) 1039.

[7] Auston D. H. in Ultrashort Light Pulses, Ed. S. L. Shapiro (Springer Verlag, Berlin) 1977.

[8] OudAR J. L. in Nonlinear Optics : Materials and Devices, Eds. C. Flytzanics and J. L. Oudar (Springer Verlag, Berlin) 1986.

[9] Treacy E. B., Phys. Lett. 28A (1968) 34.

[10] Gomez A. S. L., Gaweia-Neto A. S. and TAYlor J. R., Opt. Quant. Elec. 20 (1988) 95.
[11] JANSky J., Corradi G. and Gyuzalian R. N., Opt. Commun. 23 (1977) 293.

[12] Salin F., Georges P., Le Saux G., Roger G. et Brun A., Revue Phys. Appl. 22 (1987) 1613.

[13] Salour M. M. in Picosecond Optoelectronic Devices, Ed. C. H. Lee (Academic Press, Orlando) 1984.

[14] Lasers femtosecondes et phénomènes ultra-rapides, Ed. Y. Meyer, Revue Phys. Appl. 22, n 12 (1987).

[15] Special issue on ultrafast optics and electronics, Ed. A. M. Johnson, IEEE J. Quant. Elec. QE 24 (1988) 183.

[16] Fleming G. R., Breton J. and Martin J. L., Nature 333 (1988) 190.

[17] Miller E. K. in Time domain measurements in electromagnetics (Van Nostrand Reinhold Company, New York) 1986.

[18] Wolf P. in Picosecond Electronics and Optoelectronics, Eds. G. A. Mourou, D. M. Bloom and C. H. Lee (Springer Verlag, Berlin) 1985.

[19] Rigg P. R., Carroll J. E. and ENG C., IEEE Proc. 127 (1980) 107.

[20] Iscoff R., Semicond. Int. 62 (1985). 
[21] Menzel E. and Buchanan R., Solid State Technol. 63 (1985).

[22] Jarayaman S. and Lee C. H., J. Appl. Phys. 44 (1973) 5480.

[23] Auston D. H., Appl. Phys. Lett. 26 (1975) 101.

[24] LeE C. H. in Picosecond Optoelectronic Devices, Ed. C. H. Lee (Academic Press) 1984, 1.

[25] Castagne R., Laval S. and Laval R., Electron. Lett. 12 (1976) 438.

[26] Auston D. H. in Picosecond Optoelectronic Devices, Ed. C. H. Lee (Academic Press, London) 1984.

[27] CoOper D. E., Appl. Phys. Lett. 47 (1985) 33.

[28] Mourou G. A. and Meyer K. E., Appl. Phys. Lett. 45 (1984) 492.

[29] Ketchen M. B., Grischkowsky D., Chen T. C., Chi C. C., Duling I. N., Halas N. J., Halbout J. M., KaSh J. A. and Li G. P., Appl. Phys. Lett. 48 (1986) 751.

[30] SPRIK R., Duling III I. N., ChI C. C. and GRISCHKowsky D., Appl. Phys. Lett. 51 (1987) 548.

[31] Le Fur P. and Auston D. H., Appl. Phys. Lett. 28 (1976) 21.

[32] Antonetti A., Maley M. M., Mourou G. and Orszag A., Opt. Commun. 23 (1977) 435.

[33] Mourou G. A. and Knox, W., Appl. Phys. Lett. $36(1980) 623$.

[34] Palmier J. F., Minot C., Lievin J. L., AlexanDRe F., HaRmand J. C., Dangla J., DubonChevalier C. and Ankri D., Appl. Phys. Lett. 49 (1986) 1260.

[35] Mourou G., Stancampiano C. V., Antonetti A. and Orszag A., Appl. Phys. Lett. 39 (1981) 295.

[36] Auston D. H., Cheung K. P. and Smith P. R., Appl. Phys. Lett. 45 (1984) 284.

[37] De Fonzo A. P. and Lutz C. R., Appl. Phys. Lett. 51 (1987) 212.

[38] Smith P. R., Auston D. H. and Nuss M. C., IEEE J. Quant. Elec. 24 (1988) 255.

[39] Auston D. H., Cheung K. P., Valdmanis J. A. and Kleinman D. A., Phys. Rev. Lett. 53 (1984) 1555.

[40] Zernicke F. and Midwinter J. E., Applied Nonlinear Optics (Wiley, New York) 1973.

[41] Valdmanis J. A., Mourou G. A. and Gabel C. W., Appl. Phys. Lett. 41 (1982) 211.

[42] Kolner B. H., Bloom D. M. and Cross P. S., Electronics Letters 19 (1983) 574.

[43] Valdmanis J. A., Mourou G. A. and Gabel C. W., IEEE J. Quant. Elec. QE 19 (1983) 664.

[44] Valdmanis J. A. and Mourou G. A., Laser Focus (Feb. 1986) p. 84 (March 1986) p. 96.

[45] Nees J. and Mourou G. A., Electron. Lett. 22 (1986) 919.

[46] Valdmanis J. A. and Pei S. S. in Picosecond Electronics and Optoelectronics II, Eds. F. J. Leonberger et al. (Springer Verlag, Berlin) 1987 , p. 4.

[47] Picosecond Electronics and Optoelectronics, Eds. G. A. Mourou, D. M. Bloom and C. H. Lee (Springer Verlag, Berlin) 1985.
Picosecond Electronics and Optoelectronics II, Eds. F. J. Leonberger, C. H. Lee, F. Capam and H. Morkoc (Springer Verlag, Berlin) 1987.

[48] Kolner B. H. and Bloom D. M., IEEE J. Quant. Elec. QE 22 (1986) 79.

[49] Hamond R. B., Paulter N. G., Wagner R. S. and EISEnstadt W. R., Appl. Phys. Lett. 45 (1984) 404.

[50] Grischkowsky D., Chi C. C. and Duling I. N. in Picosecond Electronics and Optoelectronics, Eds. G. A. Mouron et al. (Springer Verlag, Berlin) 1985.

[51] Halbout J. M., May P. G., Ketchen M. B., JACKel H., Li G. P., Chi C. C., SCheuermanN M. and SMYTH M. in Picosecond Electronics and Optoelectronics II, Ed. F. J. Leonbẹrger (Springer Verlag, Berlin) 1987.

[52] Weiner A. M., Lin P. S. D. and Marcus R. B., Appl. Phys. Lett. 51 (1987) 358.

[53] BoKor J., Johnson A. M., STORZ R. H. and Simpson W. M., Appl. Phys. Lett. 49 (1986) 226.

[54] May P., Halbout J. M. and Chin G., Appl. Phys. Lett. 51 (1987) 145.

[55] Bowers J. E., Barras C. A. and Mitschke F., Electron. Lett. 22 (1986) 633.

[56] Kolner B. H. and Bloom D. M., Electron. Lett. 20 (1984) 818.

[57] Weingarten K. J., Rodwell M. J. W. and BLoom D. M., IEEE J. Quant Elec. QE 24 (1988) 198.

[58] Antreasyan A., Garbinski P. A., Mattera V. D., Temkin J. H. and Abeles J. M., Appl. Phys. Lett. 51 (1987) 1097.

[59] Meyer K. E., DykaAr D. R. and Mourou G. A. in Picosecond Electronics and Optoelectronics, Eds. G. A. Mouron et al. (Springer Verlag, Berlin) 1985, p. 54.

[60] DykaAr D. R., Sobolewski R., Whitaker J. F., Hsiang T. Y., Mourou G. A., Hollis M. A., Clifton B. J., Nichols K. B., Bozler C. O. and MuRPhy R. A. in Utrafast Phenomena $V$ (Springer Verlag, Berlin) 1986.

[61] Schlafer J., Su C. B., Powazinik W. and Lauer R. B., Electron. Lett. 21 (1985) 469.

[62] SCHIMPE R., Bowers J. E. and KOCH T. L., Electron. Lett. 22 (1986) 454.

[63] Temkin H., Frahm R. E., Olsson, N. A., Burrus C. A. and McCoY R. J., Electron. Lett. 22 (1986) 1267.

[64] Eichen E. and Silleti A., J. Light. Tech. 5 (1987) 1377.

[65] Mahr H. and Hirch M. D., Opt. Commun. 13 (1975) 96.

[66] Downey P. A., Bowers J. E., TuCker R. S. and Agyekum E., IEEE J. Quant. Elec. QE 23 (1987) 1039.

[67] White I. H., Gallager D. F. G. and Osinsky M., Electron. Lett. 21 (1985).

[68] Onodera N., Ito H. and Inaba H., Appl. Phys. Lett. 43 (1983) 720.

[69] Arakawa Y., Sogawa T., Nishioka M., Tanaka M. and SaKaKi H., Appl. Phys. Lett. 51 (1987) 1295. 
[70] Wiesenfeld J. M., Tucker R. S. and Downey P. M., Appl. Phys. Lett. 51 (1987) 1307.

[71] NeEs J., Williamson S. and Mourou G., Ultrafast Phenomena VI, Eds. T. Yajima et al. (Springer Verlag) 1988.

[72] TAYlor A. J., Wiesenfeld J. M., Eisenstein G., Tucker R. S., TAlman J. R. and Koren U., Electron. Lett. 22 (1986) 61.

[73] Wiesenfeld J. M. and HeutMaker M. S., Electron. Lett. 24 (1988) 106.

[74] Heutmaker M. S., CoOK T. B., Bosacchi B. WIESENFELD J. M. and TUCKER R. S., IEEE J. Quant. Elec. QE 24 (1988) 226.

[75] Nahman N. S., Allred C. M., Andrews J. R., HoER C. A. and LAwTON R. A., Rapport NBS IR 79-1613 (1979).

[76] Valdmanis J. A. in Ultrafast Phenomena V, Eds. G. R. Fleming and A. E. Siegman (Springer Verlag, Berlin) 1986.

[77] Willson J. P., Sibbet W. and May P. G., Picosecond Phenomena III, Eds. K. B. Eisenthal et al. (Springer Verlag, Berlin) 1982.

[78] Dugay M. A. and Hansen J. W., Appl. Phys. Lett. 15 (1969) 192.

[79] SHAH J., IEEE J. Quant. Elec. QE 24 (1988) 276.

[80] ZySS J., LedouX I., BadaN J., OUdAR J. L., Etchepare J., Hulin D., Migus A. and ANtOnetTi A., Revue Phys. Appl. 22 (1987) 1229.

[81] Sermage B., E-MRS Meeting (Les Editions de Physique, Paris), Vol. XVI (1987) p. 391.

[82] Duguay M. A. and Mattick A. T., Appl. Opt. 10 (1971) 2162.

[83] Fontaine J. J., Diels J. C., Wang C. Y. and Sallaba H., Opt. Lett. 6 (1981) 405.

[84] Fujimoto J. G., De Silvestri S., Ippen E. P., Puliafito C. A., Margolis R. and Oseroff A., Opt. Lett. 11 (1986) 150.

[85] Cornillault J. dans Le laser, principes et techniques d'application (Lavoisier, Paris) (1986) 279.

[86] Chow W. W., Gea-Banacloche J., Pedrotti L. M., SANDERS V. E., SCHLEICH W. and Scully M. O., Rev. Mod. Phys. 57 (1985) 61.

[87] Roland J. J. and Agravail G. P., Opt. Laser Tech. (1981) 239.

[88] Aronowitz F. in Laser Applications, Ed. M. Ross (Academic Press) 1971.

[89] Martin G. J., IEEE Spectrum (1986) p. 48.

[90] Buholz N. and Chodorow M., IEEE J. Quant. Elec. QE 3 (1967) 454.

[91] Chesnoy J., A paraître.

[92] Bowers J. E., Hemenway B. R., Gnauck A. H. and WILT D. P., IEEE J. Quant. Elec. QE 22 (1986) 833.

[93] Korotky S. K., Eisenstein G., Tucker R. S., Vaselka J. J. and Raybon G., Appl. Phys. Lett. 50 (1987) 1631.

[94] Korotky S. K., Eisenstein G., TUCKer R. S., Veselka J. J. and RAybon G. in Picosecond Electronics and Optoelectronics II, Eds. F. J. Leonberger et al. (Springer Verlag, Berlin) 1987.
[95] Dolfi D. W., Nazarathy M. and Jungerman R. L., Electron. Lett. 24 (1988) 528.

[96] ShOlley M. and Nichols A., Digest IEEE MTTSymposium (1986).

[97] Cooper D. E., Appl. Phys. Lett. 47 (1985) 33.

[98] Ainslie B. J. and DAY C. R., J. Light. Tech. LT 4 (1986) 967.

[99] Gnauck A. H., Bowers J. E. and Campbell J. C., Electron. Lett. 22 (1986) 600.

[100] HeidemanN R., Scholz V. and Wedding B. Electron. Lett. 23 (1987) 1030.

[101] TUCKer R. S., Eisenstein G., Korotky S. K., Koren U., RAybon G., Veselka J. J., BUHL L. L., KASPer B. L. and Alferness R. C., Electron. Lett. 23 (1987) 208.

[102] TuCKer R. S., Eisenstein G., KorotKy S. K., Buhl L. L., Veselka J. J., Raybon G., KASPER B. L. and Alferness R. C., Electron. Lett. 23 (1987) 1127.

[103] Saruwatari M., Nakagawa K., Kawanishi S. and TAKADA $A$. in Ultrafast Phenomena VI, Ed. T. Yajima et al. (Springer Verlag, Berlin) 1988.

[104] Eisenstein G., Tucker R. S., Koren U. and KOROTKY S. K., IEEE J. Quant. Elec. QE 22 (1986) 142.

[105] Hill G. R. and Stanley I. W., Opt. Eng. 26 (1987) 349.

[106] Hasegawa A., Opt. Lett. 8 (1983) 650.

[107] Mollenauer L. F., Phil. Trans. R. Soc., London A 315 (1985) 437.

[108] StOlEn R. H. in Optical Fiber Telecommunications, Eds. S. E. Miller and A. G. Chynoweth (Academic Press, New York) 1979.

[109] Mollenauer L. F., Opt. News 42 (1986).

[110] Hasegawa A., Appl. Opt. 23 (1984) 3302.

[111] Mollenauer L. F., Stolen R. H. and Islam M. M., Opt. Lett. 10 (1985) 229.

[112] Blow K. J. and DoraN N. J., Electron. Lett. 19 (1983) 429.

[113] Mollenauer L. F. and Smith K., Opt. Lett. 13 (1988) 675.

[114] GaRdiol F., Hyperfréquences (Dunod, Presses Polytechniques romandes) 1987.

[115] LipPENS D., FrisCourt M. R., Rolland P. A. and Crosnier Y., Revue Phys. Appl. 22 (1987) 1433.

[116] Herczfeld P. R., Daryoush A. J., Rosen A., Stabile P. and Contarino V. M., $R . C$. $A$. Rev. 46 (1986) 528.

[117] Forrest J. R., Ann. Telecommun. 42 (1987) 135.

[118] Eds. O. G. Kramer and P. Siorak, High frequency optical communications, Proc. SPIE 716 (1986).

[119] Platte W., Glocker R. and Brand H., Electron. Lett. 20 (1984) 608.

[120] UHDE K., Electron. Lett. 23 (1987) 1156.

[121] Lee C. H., MAK P. S. and DE Fonzo A. P., IEEE J. Quant. Elec. QE 16 (1980) 277.

[122] Paulus P., Brinker W. and Jager D., IEEE $J$. Quant. Elec. QE 22 (1986) 108.

[123] LEE C. H. and MATHUR V. K., IEEE J. Quant. Elec. QE 17 (1981) 2098. 
[124] Clark C. J., Chauchard E. A., Webb K. J., ZaKi K., LeE C. H., PolaK-Dingels P., Hung H. A. and Huang H. C. in Picosecond Electronics and Optoelectronics II, Eds. F. J. Leonberger et al. (Springer Verlag, Berlin) 1987.

[125] Foyt A. G. and LeOnberger F. J. in Picosecond Optoelectronic devices, Ed. C. H. Lee (Academic Press, Orlando) 1984.

[126] LAM D. K. W. and MAC Donald R. I., IEEE Trans. Electron. Devices ED 31 (1984) 1766.

[127] Mourou G., Stancampiano C. V. and Blumenthal D., Appl. Phys. Lett. 38 (1981) 470.

[128] Salles A. A. and Forrest J. R., Appl. Phys. Lett. 38 (1981) 392.

[129] KøPF G. A., SPIE Proc. 477 (1986) 75.

[130] PAN J. J., SPIE Proc. 716 (1986) 23.

[131] Gee C. M., Newberg I. L., Thurmond G. D. and YEN H. W., SPIE Proc. 716 (1986) 64.

[132] Hsu H. P., De la Chapelle M. and Gulick J. J., SPIE Proc. 716 (1986) 69.

[133] Suematsu Y., Arai S. and Kishino K., J. Light. Tech. LT 1 (1983) 161.

[134] Goldberg L., TAYlor H. F., Weller J. F. and Bloom D. M., Electron. Lett. 19 (1983) 491.

[135] Goldberg L., Yurek A. M., Weller J. F. and TAYLOR H. F., Electron. Lett. 21 (1985) 814.
[136] VAN DER ZIEL J. P. in Semiconductors and semimetals (Academic Press) Vol. 22 (1985) p. 1.

[137] Kuhl J., Serenyi M. and Gobel E. O., Opt. Lett. 12 (1987) 334.

[138] Corzine S. W., Bowers J. E., Przybylek G., Koren U., Miller B. I. and SocCOlich C. E. Appl. Phys. Lett. 52 (1988) 348.

[139] Silberberg Y. and SMITH P. W., IEEE J. Quant. Elec. QE 22 (1986) 759.

[140] Akiba S., Williams G. E. and Haus H. A., Electron. Lett. 17 (1981) 527.

[141] Lau K. Y., Ury I. and Yariv A., Appl. Phys. Lett. 46 (1985) 1117.

[142] Tucker R. S., Koren V., Raybon G., Burrus C. A., Miller B. I., KOCH T. L., Eisenstein G. and SHAHAR A., IEEE International Semiconductor Laser Conf., Boston (1988).

[143] L'optique dans l'ordinateur, Eds. P. Chavel, S. Laval, J. Taboury, Revue Phys. Appl. 22 (1987) 1215

[144] Hulin D., Antonetti A., Joffre M., Migus A., MYSYROWICZ A., PEYGAMBARIAN N. and Gibis H. M., Revue Phys. Appl. 22 (1987) 1269.

[145] Photonic Switching, Eds. T. K. Gustafson and P. W. Smith (Springer Verlag, Berlin) 1988. 